\title{
Viscoelastic Properties of Polymer-Grafted Nanoparticle Composites from Molecular Dynamics Simulations
}

\author{
Gregory D. Hattemer and Gaurav Arya* \\ Department of NanoEngineering, University of California, San Diego, 9500 Gilman Drive, Mail Code 0448, La Jolla, California \\ 92093, United States
}

\section{Supporting Information}

ABSTRACT: To provide insights into how polymer-grafted nanoparticles (NPs) enhance the viscoelastic properties of polymers, we have computed the frequency-dependent storage and loss modulus of coarse-grained models of polymer nanocomposites by means of molecular dynamics simulations. Nanocomposites containing NPs grafted with chains similar to those comprising the host polymer matrix exhibit considerably higher moduli than nanocomposites containing bare NPs across the entire frequency range investigated. This effect is shown to arise from the additional distortion of the shear field
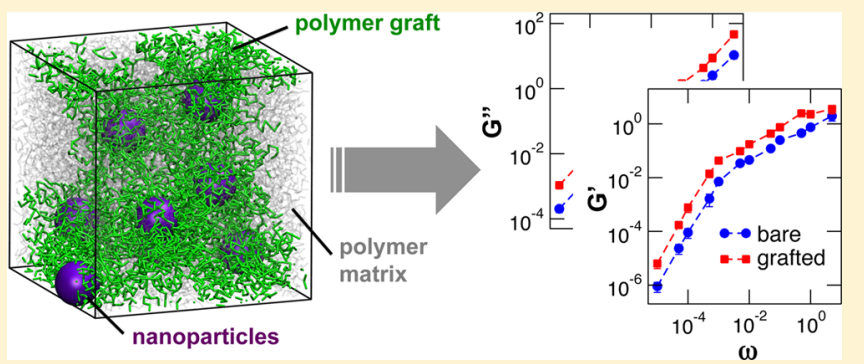
in the polymer matrix resulting from the grafted chains and from the slower relaxation time of the grafted chains compared to the matrix chains when the former are at least half as long as the latter. Increasing the attraction between the grafted and matrix chains results in further enhancement in the two moduli, but only at frequencies slower than those corresponding to the longest relaxation time of the chains. This effect is shown to arise from a dramatic slowdown in the relaxation dynamics of both the matrix and grafted chains. In addition, the nanocomposite moduli are found to increase with decreasing NP size and increasing NP loading, grafted chain length, and grafting density with varying frequency dependence. These parametric effects are also explained in terms of shear distortion effects and chain relaxation times. Based on these results, a phenomenological model is proposed to estimate the storage and loss modulus of such nanocomposites as a function of the Rouse relaxation times of the grafted and matrix chains and the volume fractions of the NPs, grafted chains, and matrix chains. The model captures the observed dependence of the moduli with the examined parameters of the grafted NPs and yields moduli predictions that agree quantitatively with those computed from the simulations at low frequencies.

\section{INTRODUCTION}

The incorporation of nanoparticles (NPs) into polymer can drastically alter the properties of the host polymer. ${ }^{1,2}$ The nanoscopic size of NPs allows for high particle number densities to be achieved at small particle loadings $<10 \mathrm{wt} \%$, enabling a large fraction of the polymer to be in direct contact with the surface of the particles. ${ }^{2,3}$ The NPs are often grafted with polymer chains to introduce steric (entropic) repulsion between the NPs and prevent their aggregation due to attractive van der Waals and depletion forces. ${ }^{4,5}$ The ability to integrate particles with different properties-size, shape, stiffness, and interactions-offers an easy and highly versatile approach for tuning polymer properties. Indeed, such polymer nanocomposites (PNCs) have attracted a lot of attention recently and have found many applications in industry. ${ }^{6-8}$

An important class of properties relevant to PNC characterization, processing, and applications are the viscoelastic properties: the time $(t)$-dependent stress relaxation spectrum $G(t)$, and its Fourier transforms, the frequency $(\omega)$-dependent storage modulus $G^{\prime}(\omega)$, and loss modulus $G^{\prime \prime}(\omega)$. Much effort has been devoted to understanding the effects of NPs on the viscoelastic properties of polymers and their relation to the underlying structure and dynamics of the polymer..$^{9-16}$ Most notably, the addition of NPs enhances the $G^{\prime}(\omega)$ and $G^{\prime \prime}(\omega)$ of the polymers in systems as diverse as, for example, carbon nanotubes in polyethylene, ${ }^{9}$ silica spheres in poly(ethylene oxide), ${ }^{10}$ and layered silicates in polystyrene-polyisoprene copolymers. ${ }^{11}$ The enhancement in the two moduli is usually more pronounced at low frequencies and stronger in $G^{\prime}(\omega)$ compared to $G^{\prime \prime}(\omega)$. In fact, at sufficiently high NP loadings, ranging from $\sim 2$ to $15 \mathrm{wt} \%$ depending on the system, the $G^{\prime}(\omega)$ and $G^{\prime \prime}(\omega)$ profiles begin to plateau at low frequencies, reminiscent of more solid-like behavior. ${ }^{10,11}$ Such effects have been observed in both bare ${ }^{9,10}$ and polymer-grafted NPs. ${ }^{11,12}$

The observed enhancement in the viscoelastic properties of PNCs has been attributed to various physical effects that have been examined using theory and simulations. The solid-like response in $G^{\prime}(\omega)$ and $G^{\prime \prime}(\omega)$ observed at high loadings was initially thought to be due to jamming of particles, especially given that several of the particles or particulate phases being studied were highly anisotropic. ${ }^{12,13}$ However, calculations indicated that the percolation threshold would be approached

Received: October 15, 2014

Revised: January 6, 2015

Published: February 10, 2015 
at loadings 5-10 times larger than those signifying the onset of the solid-like response, ${ }^{10,17}$ suggesting that the range of influence of nanoparticles goes well beyond their boundaries. Indeed, it is now well-known that a significant slowdown in the dynamics of the polymer chains occurs due to their strong confinement at high particle loadings ${ }^{18}$ and due to the adsorption of polymer chains at the surface of the NPs when the chains exhibit strong interactions with the surface. ${ }^{11,12}$ This effect has been shown to dramatically enhance the viscoelastic properties of the host polymer in various coarse-grained simulations of bare-NP/polymer systems..$^{15,17,19}$ Some enhancement in $G^{\prime}(\omega)$ and $G^{\prime \prime}(\omega)$ must also arise from particleinduced distortion of the strain field within the polymer ${ }^{17,20}$ caused by constraints imposed on polymer mobility at its interface with the particle, e.g., no-slip boundary condition, leading to enhanced viscous dissipation and stiffness. This effect has been considered in continuum models of suspension viscosity $^{21}$ and composite modulus. ${ }^{20}$

While the above studies have greatly enhanced our understanding of the viscoelastic behavior of PNCs, how polymer chains grafted onto NPs affect the viscoelastic properties of the composites is not fully understood. How these effects depend on various attributes of the NPs like their size, shape, graft length, and grafting density also remains unknown. Many of the PNCs studied thus far have in fact contained polymer-grafted NPs, but a systematic study of differences in the viscoelasticity of PNCs containing grafted versus bare NPs is missing. Similarly, theoretical and simulation studies on the viscoelastic behavior of PNCs have considered only bare NPs, ${ }^{15,17,19}$ and studies that did model polymergrafted NPs focused on other aspects like NP dynamics, interactions, aggregation, and phase behavior, which are reviewed in several recent articles. ${ }^{2,3,22-24}$ Understandably, polymer grafting introduces an additional layer of complexity to an already complex problem of predicting properties of PNCs based on properties of its constituents. Nevertheless, it is expected that the grafted chains would extend the range of influence of the NPs by penetrating into the host polymer and also interpenetrating with the grafts on neighboring NPs. Indeed, it was recently shown that the grafting of silica nanoparticles with long polyethylene chains facilitates the formation of sheet-shaped gel-like domains of NPs that provides additional reinforcement to the PNCs. ${ }^{14}$ Moreover, the grafted chains, as a result of their attachment to NPs through one of their ends, exhibit dynamics that are fundamentally different from those of the free matrix chains, but it is not clear how these dynamics would affect stress relaxation in the PNCs.

Here we provide new insights into the viscoelastic properties of PNCs containing polymer-grafted NPs by modeling the PNCs via simple, coarse-grained models and computing their frequency-dependent storage and loss moduli from exhaustive molecular dynamics simulations. Our calculations show that PNCs containing grafted NPs yield significantly higher $G^{\prime}(\omega)$ and $G^{\prime \prime}(\omega)$ than those containing bare NPs and that both moduli increase with decreasing NP size and increasing NP loading, grafting density, graft length, and graft/matrix affinity. We show how these parametric changes lead to varying extents of shear distortion and changes in chain relaxation times, yielding rich viscoelastic behavior. We conclude by proposing a simple phenomenological model that provides quantitative estimates of $G^{\prime}(\omega)$ and $G^{\prime \prime}(\omega)$ to within a constant scaling factor. Given that grafted chains offer an additional, versatile parameter for modifying the polymer/NP interactions in PNCs for tuning their properties, this study should be relevant to the design and optimization of such PNCs for targeted applications.

\section{COMPUTATIONAL METHODS}

Coarse-Grained Model. To elucidate the role of NP grafts on the viscoelastic properties of PNCs, we choose a simple coarse-grained representation of the host polymer, grafted polymer, and NPs. Such a representation allows computation of viscoelastic properties-which are notoriously difficult to compute from simulations-within reasonable computational costs while still capturing the essential physics of polymer conformational dynamics. Using simplified models also allows us to keep our observations sufficiently generic and applicable to a broad range of systems. An atomistic treatment of PNCs is currently not feasible anyway due to the large system sizes and sluggish dynamics of the NPs and the polymer chains.

Our model PNC system is composed of polymer-grafted spherical NPs within a linear polymer (Figure 1a),

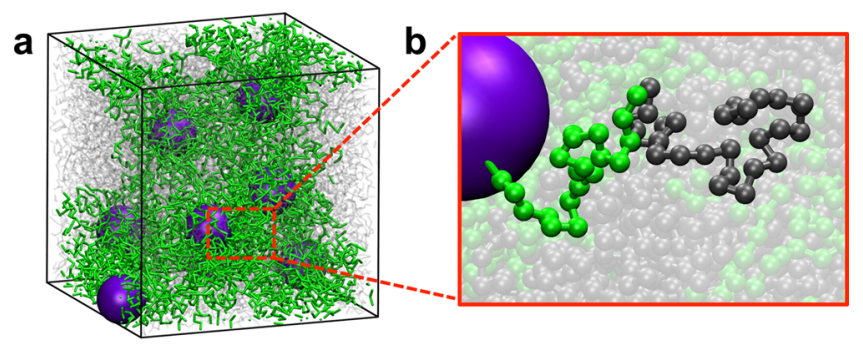

Figure 1. (a) Simulation setup employed for examining the viscoelasticity of polymer/nanoparticle systems. The nanoparticles are colored violet while the matrix and grafted chains are shown as gray and green tubes, respectively. (b) Close-up view of the simulation system emphasizing the models of polymer-grafted nanoparticles and polymer matrix employed in this study. For visual clarity, we show only a single grafted and matrix chain in opaque color, while the remaining chains are shown in transparent colors.

representative of several experimental systems studied before. $^{10,14} \mathrm{We}$ model the host polymer matrix using the popular bead-chain model of Kremer and Grest ${ }^{25}$ (Figure 1b). In this model, segments of the polymer matrix are treated as beads of size $\sigma$ and mass $m$. The adjacent beads in each matrix chain, of length $L_{\text {matrix }}$ beads, are connected via a strong finitely extensible nonlinear elastic (FENE) potential:

$$
U_{\mathrm{FENE}}=-\frac{k}{2} R_{0}{ }^{2} \ln \left[1-\left(\frac{r}{R_{0}}\right)^{2}\right]
$$

where $r$ is the distance between bonded beads, $R_{0}=1.5 \sigma$ is the maximum possible length of the spring, $k=30 \varepsilon / \sigma^{2}$ is the spring constant, and $\varepsilon$ sets the energy scale of the system (see below). Furthermore, all nonbonded interactions between polymer matrix beads, both intra- and intermolecular, are treated using a short-range purely repulsive potential, also known as the Weeks-Chandler-Anderson (WCA) potential: ${ }^{26}$

$$
U_{\mathrm{WCA}}= \begin{cases}4 \varepsilon\left[\left(\frac{\sigma}{r}\right)^{12}-\left(\frac{\sigma}{r}\right)^{6}+\frac{1}{4}\right] & r<2^{1 / 6} \sigma \\ 0 & r \geq 2^{1 / 6} \sigma\end{cases}
$$

where $r$ is the distance between the nonbonded beads. 
The polymer-grafted NPs are also treated using a coarsegrained representation (Figure $1 \mathrm{~b}$ ). The cores of the NPs are treated as spheres of diameter $d_{\mathrm{NP}}$ and mass $m_{\mathrm{NP}}$ that interact with each other via a distance-shifted Lennard-Jones (LJ)

potential:

$$
U_{\text {shifted-LJ }}= \begin{cases}4 \varepsilon\left[\left(\frac{\sigma}{r-r_{\mathrm{ev}}}\right)^{12}-\left(\frac{\sigma}{r-r_{\mathrm{ev}}}\right)^{6}-\left(\frac{\sigma}{r_{\mathrm{c}}-r_{\mathrm{ev}}}\right)^{12}+\left(\frac{\sigma}{r_{\mathrm{c}}-r_{\mathrm{ev}}}\right)^{6}\right] & r-r_{\mathrm{ev}}<r_{\mathrm{c}} \\ 0 & r-r_{\mathrm{ev}} \geq r_{\mathrm{c}}\end{cases}
$$

where $r$ is the distance between the centers of the interacting NPs, $r_{\mathrm{ev}}=d_{\mathrm{NP}}-\sigma$ to prevent the NPs from interpentrating each other, and $r_{\mathrm{c}}=2.5 \sigma$ is the cutoff distance of the potential. The NP-grafted polymer chains are also treated using the Kremer-Grest model, i.e., their adjancent beads are connected via FENE springs (eq 1), and nonbonded interactions between the beads are modeled via the WCA potential (eq 2). The grafted chains, each of length $L_{\text {graft }}$ beads, are attached to the surface of bare NPs using FENE springs (eq 1). The number of grafted chains per nanoparticle $n_{\text {graft }}$ is determined from the grafting density $\Gamma_{\text {graft }}$ according to $n_{\text {graft }}=\Gamma_{\text {graft }} \times \pi d_{\mathrm{NP}}{ }^{2}$. The grafting points are distributed uniformly across the NP surface by using the "generalized spiral points" algorithm of Rakhmanov et al. ${ }^{27,28}$ These grafting points are held fixed relative to each other by treating them as "virtual" beads that are held fixed relative to their NP center using rigid body constraints. The interactions between the NPs and the grafted/ matrix chains are also treated using the distance-shifted LJ potential (eq 3 ) with a separate set of distance shifts $r_{\mathrm{ev}}=\left(d_{\mathrm{NP}}\right.$ $-\sigma) / 2$ for each NP size to prevent overlap between NPs and polymer chains. The nonbonded intermolecular interactions between the grafted chains and between the grafted and matrix chains are also treated via the WCA potential (eq 2).

To investigate the effect of favorable interactions between the grafted and matrix chains, we introduce additional attractive interactions between $n_{\text {att }}$ terminal beads of the grafted chains and the same number of centrally located and terminal beads of the matrix chains. Hence, $n_{\text {att }}=0$ describes "neutral" interactions and $n_{\text {att }}>0$ describe "attractive" interactions between the two chain types, with the strength of the attraction increasing with increasing values of $n_{\text {att }}$. Such attractive interactions mediated between only specified beads of the chains is chosen to mimic favorable interactions between periodically spaced chemical groups found on many functional polymers. One such polymer is polyurea that is currently being studied as part of the polymer matrix and an NP graft in the context of shockmitigating PNCs. ${ }^{29}$ Polyurea, is an alternating copolymer composed of repeating units of long, aliphatic segments and short, rigid aromatic moieties flanked by urea groups. $^{30,31}$ The attractive beads in our model could then represent the aromatic moeties in polyurea that interact strongly with each other across chains due to hydrogen bonding and stacking interactions, and the remaining repulsive beads in our model chains could represent the long aliphatic segments in polyurea that interact weakly with each other. We model the attractive interaction between the specified beads of the grafted and matrix chains via a short-range attractive potential: $:^{30,32}$

$$
U_{\mathrm{att}}= \begin{cases}4 \varepsilon\left[\left(\frac{\sigma}{r}\right)^{12}-\left(\frac{\sigma}{r}\right)^{6}+\frac{1}{4}\right]-\Phi & r<2^{1 / 6} \sigma \\ \frac{1}{2} \Phi\left[\cos \left(\alpha r^{2}+\beta\right)-1\right] & 2^{1 / 6} \sigma \leq r<1.5 \sigma \\ 0 & r \geq 1.5 \sigma\end{cases}
$$

where the constants $\alpha=3.17307$ and $\beta=-0.85623$ are chosen to ensure that the potential is continuous and approaches smoothly to zero at the cutoff distance and $\Phi$ sets the magnitide of the attractive interactions. According to our previous work on polyurea, ${ }^{30}$ we set $\Phi=2.5 \varepsilon$.

All simulation parameters and quantities are henceforth reported in units of $\sigma, m$, and $\varepsilon$, which set the respective length, mass, and time scales in this study.

Simulation Details and Analyses. The viscoelastic properties of the grafted NP-polymer matrix are computed from equilibrium molecular dynamics (MD) simulations in the canonical ensemble. We use the LAMMPS package developed by Sandia National Laboratories ${ }^{33}$ for carrying out the simulations. The equations of motion are integrated using a velocity-Verlet algorithm with a time step of $\Delta t=0.005$, and the temperature is kept fixed via a Nosé-Hoover thermostat. ${ }^{34}$ Consistent with our previous studies, ${ }^{30}$ we set the temperature $T=\varepsilon / k_{\mathrm{B}}$ (where $k_{\mathrm{B}}$ is the Boltzmann constant) so the thermal and interaction energies are of the same magnitude. Based on previous studies, ${ }^{25}$ this temperature is expected to be roughly twice the glass transition temperature $T_{\mathrm{g}}$ of the polymer systems studied here. Because of computational limitations in sampling chain conformations at temperatures equal to or below $T_{\mathrm{g}}$, our simulations are limited to temperatures well above $T_{\mathrm{g}}$.

The simulations are started from a nonoverlapped configuration of $n_{\mathrm{NP}}$ grafted NPs and $n_{\text {matrix }}$ matrix chains in a large simulation box implementing periodic boundary conditions. The simulation box is gradually compressed to a volume $V$ during the initialization phase of the MD simulation until a reasonable polymer melt-like density of $\rho_{\text {polymer }} \equiv n_{\text {beads }} /$ $V_{\text {beads }}=0.82$ is obtained, where $n_{\text {beads }}$ is the total number of polymer beads in the simulation box and $V_{\text {beads }}$ is the volume of the simulation box occupied by the grafted and matrix polymer only but not the NPs. We consider the NPs to have a density of $\rho_{\mathrm{NP}}=1.9$, approximately 2.3 times larger than that of the polymer, typical of silica NPs in a polyurea matrix. ${ }^{31}$ The NP mass is then determined via $m_{\mathrm{NP}}=\rho_{\mathrm{NP}}\left(\pi d_{\mathrm{NP}}{ }^{3} / 6\right)$. We utilize large system sizes composed of $n_{\text {beads }} \simeq 30000-40000$, and each simulation is performed for 27 million time steps, where the first 1 million times steps (equilibration) are discarded.

The storage modulus $G^{\prime}(\omega)$ and the loss modulus $G^{\prime \prime}(\omega)$ are computed by converting the time-dependent shear modulus $G(t)$ into its frequency $(\omega)$-dependent form. The shear 
Table 1. System Parameters for the Different Simulations Carried Out In This Study

\begin{tabular}{|c|c|c|c|c|c|c|c|c|c|c|}
\hline \multirow[b]{2}{*}{ system no. ${ }^{a}$} & \multicolumn{6}{|c|}{ independent parameters } & \multicolumn{4}{|c|}{ dependent parameters } \\
\hline & $\mathrm{B} / \mathrm{G}^{b}$ & $d_{\mathrm{NP}}^{c}$ & $f_{\mathrm{NP}}{ }^{d}$ & $L_{\text {graft }}^{e}$ & $\Gamma_{\text {graft }}^{f}$ & $n_{\text {att }}^{g}$ & $n_{\mathrm{NP}}^{h}$ & $n_{\text {graft }}^{i}$ & $n_{\text {matrix }}^{j}$ & $V^{k}$ \\
\hline \multicolumn{11}{|c|}{ effect of NP grafting } \\
\hline 1 & B & 4.0 & 0.06 & 20 & 0.4 & 0 & 27 & 0 & 677 & 33929 \\
\hline 2 & G & 4.0 & 0.06 & 20 & 0.4 & 0 & 27 & 20 & 405 & 33832 \\
\hline 3 & B & 6.0 & 0.06 & 20 & 0.4 & 0 & 8 & 0 & 677 & 33929 \\
\hline 4 & G & 6.0 & 0.06 & 20 & 0.4 & 0 & 8 & 45 & 497 & 33929 \\
\hline 5 & B & 8.0 & 0.06 & 20 & 0.4 & 0 & 4 & 0 & 802 & 40194 \\
\hline 6 & G & 8.0 & 0.06 & 20 & 0.4 & 0 & 4 & 80 & 642 & 40194 \\
\hline \multicolumn{11}{|c|}{ effect of NP size } \\
\hline 2 & G & 4.0 & 0.06 & 20 & 0.4 & 0 & 27 & 20 & 405 & 33832 \\
\hline 4 & G & 6.0 & 0.06 & 20 & 0.4 & 0 & 8 & 45 & 497 & 33929 \\
\hline 6 & G & 8.0 & 0.06 & 20 & 0.4 & 0 & 4 & 80 & 642 & 40194 \\
\hline \multicolumn{11}{|c|}{ effect of NP loading } \\
\hline 2 & G & 4.0 & 0.06 & 20 & 0.4 & 0 & 27 & 20 & 405 & 33832 \\
\hline 7 & G & 4.0 & 0.12 & 20 & 0.4 & 0 & 27 & 20 & 52 & 16612 \\
\hline 4 & G & 6.0 & 0.06 & 20 & 0.4 & 0 & 8 & 45 & 497 & 33929 \\
\hline 8 & G & 6.0 & 0.12 & 20 & 0.4 & 0 & 16 & 45 & 274 & 32737 \\
\hline 6 & G & 8.0 & 0.06 & 20 & 0.4 & 0 & 4 & 80 & 642 & 40194 \\
\hline 9 & G & 8.0 & 0.12 & 20 & 0.4 & 0 & 8 & 80 & 431 & 38779 \\
\hline \multicolumn{11}{|c|}{ effect of graft length } \\
\hline 10 & G & 6.0 & 0.06 & 10 & 0.4 & 0 & 8 & 45 & 587 & 33929 \\
\hline 4 & G & 6.0 & 0.06 & 20 & 0.4 & 0 & 8 & 45 & 497 & 33929 \\
\hline 11 & G & 6.0 & 0.06 & 30 & 0.4 & 0 & 8 & 45 & 407 & 33929 \\
\hline 12 & G & 6.0 & 0.06 & 40 & 0.4 & 0 & 8 & 45 & 317 & 33929 \\
\hline \multicolumn{11}{|c|}{ effect of graft density } \\
\hline 13 & G & 6.0 & 0.06 & 20 & 0.1 & 0 & 8 & 12 & 633 & 33929 \\
\hline 14 & G & 6.0 & 0.06 & 20 & 0.2 & 0 & 8 & 23 & 585 & 33929 \\
\hline 4 & G & 6.0 & 0.06 & 20 & 0.4 & 0 & 8 & 45 & 497 & 33929 \\
\hline 15 & G & 6.0 & 0.06 & 20 & 0.8 & 0 & 8 & 90 & 317 & 33929 \\
\hline \multicolumn{11}{|c|}{ effect of graft/matrix affinity } \\
\hline 2 & G & 6.0 & 0.06 & 20 & 0.4 & 0 & 8 & 45 & 497 & 33929 \\
\hline 16 & G & 6.0 & 0.06 & 20 & 0.4 & 1 & 8 & 45 & 497 & 33929 \\
\hline 17 & $\mathrm{G}$ & 6.0 & 0.06 & 20 & 0.4 & 2 & 8 & 45 & 497 & 33929 \\
\hline
\end{tabular}

${ }^{a}$ Index identifying different simulation systems from a total of 15 systems that were examined. ${ }^{b}$ "B" signifies bare NPs, while " $G$ " signifies grafted NPs. ${ }^{c}$ NP diameter. ${ }^{d}$ Weight fraction of NPs. ${ }^{e}$ Length of grafted chains. ${ }^{f}$ Surface grafting density. ${ }^{g}$ Number of adjacent, mutually attractive beads on grafted and matrix chains. ${ }^{h}$ Number of NPs in the simulation box. ${ }^{i}$ Number of grafted chains per NP. ${ }^{j}$ Number of matrix chains in the simulation box. ${ }^{k}$ Volume of the simulation box.

modulus $G(t)$ is computed from the stress autocorrelation function (SACF):

$$
G(t)=\frac{V}{k_{\mathrm{B}} T}\left\langle\sigma_{\alpha \beta}(t) \sigma_{\alpha \beta}(0)\right\rangle
$$

where $V$ is the volume of the simulation box, $\langle\cdots\rangle$ denotes ensemble average, and $\sigma_{\alpha \beta}$ is the $\alpha-\beta$ component of the stress tensor (where $\alpha \neq \beta$ ), calculated via the virial theorem:

$$
\sigma_{\alpha \beta}=\frac{1}{V}\left[\sum_{i=1}^{n_{\text {beads }}} m_{i} v_{i \alpha} v_{i \beta}+\sum_{i=1}^{n_{\text {beads }}-1} \sum_{j=i+1}^{n_{\text {beads }}} r_{i j \alpha} F_{i j \beta}\right]
$$

where $m_{i}, v_{i \alpha}$ and $v_{i \beta}$ are the mass and $\alpha$ - and $\beta$-component velocities of bead $i$, respectively, and $r_{i j \alpha}$ and $F_{i j \beta}$ are the $\alpha$ component separation distance and $\beta$-component force acting between beads $i$ and $j$, respectively. Since the three off-diagonal elements of the stress tensor $\sigma_{x y}, \sigma_{x z}$ and $\sigma_{y z}$ are equivalent, we use the average of the individual SACFs obtained from the three stresses to obtain smoother estimates of the SACF. Furthermore, the stresses are recorded every time step to obtain accurate results, as done in previous studies. ${ }^{30}$
The frequency-dependent complex shear modulus $G^{*}(\omega)$ is then calculated as the Fourier transform of $G(t)$ :

$$
G^{*}(\omega)=i \omega \int_{0}^{\infty} \mathrm{e}^{-i \omega t} G(t) \mathrm{d} t
$$

where $G^{\prime}(\omega)$ and $G^{\prime \prime}(\omega)$ are the real and complex components of $G^{*}(\omega)$, respectively:

$$
\begin{aligned}
& G^{\prime}(\omega)=\omega \int_{0}^{\infty} G(t) \sin (\omega t) \mathrm{d} t \\
& G^{\prime \prime}(\omega)=\omega \int_{0}^{\infty} G(t) \cos (\omega t) \mathrm{d} t
\end{aligned}
$$

The dissipative factor $\tan \delta$ is obtained as $G^{\prime \prime} / G^{\prime}$ but is not reported here due to large uncertainties that prevent any trends to be gleaned. To obtain reliable estimates and uncertainties in the viscoelastic moduli, we perform $n_{\text {runs }}=4$ independent simulation runs, each starting from a unique starting configuration, for each PNC system investigated here. The reported storage and loss moduli are calculated as the mean of the four $G^{\prime}(\omega)$ and $G^{\prime \prime}(\omega)$ calculated from the runs, and the reported uncertainties (error bars) in the moduli are calculated 
as the standard error of the means (SEM), i.e., standard deviation of the four $G^{\prime}(\omega)$ and $G^{\prime \prime}(\omega)$ obtained from the runs divided by $\sqrt{ } n_{\text {runs }}$

Parametric Study. To investigate how polymer-grafted NPs modulate the viscoelastic properties of the host polymer, we examine the effects of six parameters associated with the NPs, namely, NP grafting (bare vs grafted), NP diameter $d_{\mathrm{NP}}$, NP loading $f_{\mathrm{NP}}$ (defined as weight \% of NPs in the PNC), grafted chain length $L_{\text {graft }}$ grafting density $\Gamma_{\text {graft }}$ and strength of attractive graft/matrix interactions characterized by $n_{\text {att }}$. To keep the list of variable parameters down to a reasonable number, we fix all parameters not directly related to the grafted NPs, such as the matrix chain length that is set to $L_{\text {matrix }}=40$, the polymer density and temperature that are set to $\rho_{\text {polymer }}=$ 0.82 and $T=1$, and the interactions among the NPs and the matrix chains that are set via eqs 1 and 2 .

To dissect the effects of the parameters listed above, we carry out different groups of simulations, each examining the effects of a chosen parameter by varying it across physically and computationally accessible bounds while keeping the remaining parameters constant (Table 1). Specifically, to elucidate the effects of NP grafting, we simulate PNCs with bare and grafted NPs keeping other parameters fixed across the two sets of simulations. To elucidate the effects of NP size, we explore $d_{\mathrm{NP}}$ in the range 4-8 comparable to the size of the matrix chains ( $\sim 2 R_{\mathrm{g}} \approx 6.32$, where $R_{\mathrm{g}}$ is the radius of gyration of the chains). To elucidate the effects of NP loading, we vary $f_{\mathrm{NP}}$ in the range $6-12 \mathrm{wt} \%$, corresponding to NP volume fractions in the range 0.027-0.054 well below the dense-packing regime. To elucidate the effect of graft length, we vary $L_{\text {graft }}$ in the range $10-40$, i.e., spanning lengths much shorter than the matrix chains to those comparable to the matrix chains. To elucidate the effect of grafting density, we vary $\Gamma_{\text {graft }}$ within $0.1-0.8$ (chains $/ \sigma^{2}$ ) spanning sparse to dense grafting regimes. Finally, to elucidate the effects of graft/matrix interactions, we vary the number of mutually attractive beads $n_{\text {att }}$ from 0 to 2, signifying neutral, weakly attractive, and strongly attractive systems. Note that to keep $f_{\mathrm{NP}}$ and $\Gamma_{\text {graft }}$ constant in simulations exploring effects of other parameters, the total number of NPs in the simulation box $n_{\mathrm{NP}}$ and the number of grafted chains per NP $n_{\text {graft }}$ are adjusted accordingly. Additionally, to keep $\rho_{\text {polymer }}$ fixed across the different systems, we adjust the number of matrix chains $n_{\text {matrix. }}$ Therefore, systems with larger NP loadings, which naturally result in a larger number of grafted chains, consequently result in a smaller number of matrix chains $n_{\text {matrix }}$ to maintain $\rho_{\text {polymer }}$ constant. By same reasoning, the bare NP systems contain a larger number of matrix chains than the grafted NPs.

To quantify the extent of crowding of grafted chains at the NP surface, we have calculated a dimensionless grafting density $\Gamma^{*} \equiv \Gamma_{\text {graft }} R_{\mathrm{g}, \mathrm{b}}{ }^{2}$, where $R_{\mathrm{g}, \mathrm{b}}$ is the radius of gyration of an "unperturbed" grafted chain, i.e., if it was not grafted to the NP surface but was present free in the polymer matrix. ${ }^{35} \Gamma^{*}$ thus represents the grafting density in units of number of grafted chains per characteristic surface area of the chains. Table $S 1$ in the Supporting Information tabulates $R_{\mathrm{g}, \mathrm{b}}$ and the resulting $\Gamma^{*}$ for each of the systems studied here. We note that $\Gamma^{*}$ varies between 0.48 (mushroom regime) and 3.8 (stretched brush regime) as we vary the grafting density from $\Gamma_{\text {graft }}=0.1$ to 0.8 . Similarly, $\Gamma^{*}$ varies from 0.86 to 3.98 as we vary the grafted chain length from $L=10$ to 40 . Thus, we probe similar crowding regimes when examining the effects of grafting density and chain length.
To confirm that none of the PNC systems studied here exhibit any NP aggregation, which would otherwise mask the effects of the parameters being investigated above, we have carried our various tests involving calculation of inter-NP radial distribution function and use of thumb rules suggested by Kumar et al. ${ }^{36}$ A detailed discussion of these tests is provided in Section S1 and Figure S1 of the Supporting Information. We have also calculated the translational and rotational relaxation times of the NPs to investigate whether our simulations are sufficiently long to capture equilibrium behavior of each of our PNCs, which are expected to exhibit sluggish dynamics inspite of $T>T_{\mathrm{g}}{ }^{37}$ Our results, tabulated in Table S2 and discussed in Section S2 of the Supporting Information, suggest that our simulations times of $>100000$ should be able to fully capture the entire stress relaxation spectrum for most of the examined PNC systems except the system with strongly attractive interactions $\left(n_{\text {att }}=2\right)$, which would require prohibitively long simulations.

\section{RESULTS AND DISCUSSION}

Effect of NP Grafting. We begin by analyzing differences in the viscoelasticity of PNCs containing bare NPs versus those grafted with neutral chains. Figure 2 plots the computed $G^{\prime}(\omega)$

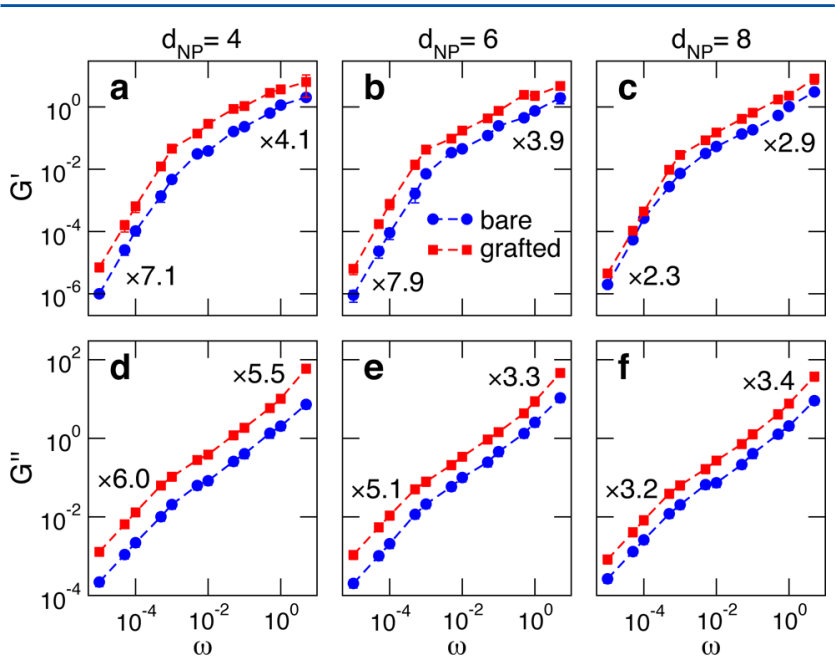

Figure 2. Comparison of the storage modulus $G^{\prime}(\mathrm{a}-\mathrm{c})$ and the loss modulus $G^{\prime \prime}(\mathrm{d}-\mathrm{f})$ of polymers containing grafted NPs (red squares) versus those containing bare NPs (blue circles) of size $d_{\mathrm{NP}}=4(\mathrm{a}, \mathrm{d})$, $d_{\mathrm{NP}}=6(\mathrm{~b}, \mathrm{e})$, and $d_{\mathrm{NP}}=8(\mathrm{c}, \mathrm{f})$. The fold enhancement in the two moduli at low and high frequencies is also indicated.

and $G^{\prime \prime}(\omega)$ for polymer matrices with grafted and bare NPs of size $d_{\mathrm{NP}}=4,6$, and 8 . The results show that the tethering of polymers onto NPs leads to a substantial increase in both $G^{\prime}(\omega)$ (Figure $2 \mathrm{a}-\mathrm{c}$ ) and $G^{\prime \prime}(\omega)$ (Figure $2 \mathrm{~d}-\mathrm{f}$ ) across the entire frequency range. A similar increase in $G^{\prime}(\omega)$ and $G^{\prime \prime}(\omega)$ with grafting has also been observed in experimental studies on silica NP-based polymer composites. ${ }^{10}$ Furthermore, we find that the $G^{\prime}(\omega)$ and $G^{\prime \prime}(\omega)$ profiles of grafted NPs look similar in shape to those of the bare NPs, except for their upward shift in the log-log plot, suggesting that both moduli increase by an almost constant factor across all frequencies upon grafting. To better quantify this enhancement, we have calculated the fold enhancement in the two moduli at low and high frequencies, averaged over frequencies in the range $\omega \in$ [0.00001-0.0005] and $\omega \in[0.1-1]$, respectively. These ratios, indicated in Figure 2 , show that the enhancement is slightly larger at low 
frequencies, especially for $G^{\prime}$ and for $d_{\mathrm{NP}}=4$ and 6 . We also note that the location of the inflection point in the $G^{\prime}(\omega)$ profiles, typically associated with the slowest relaxation time of the polymer, seems to be largely unaffected by grafting, though there seems to be a slight shift toward low frequencies. The above observations suggest that the grafting-induced enhancement in $G^{\prime}(\omega)$ and $G^{\prime \prime}(\omega)$ observed here likely results from a frequency-dependent and frequency-independent mechanism and that the latter might be more dominant.

To investigate the effect of NP grafting on the dynamics of the matrix chains, we have computed the Rouse relaxation time for matrix chains in the bare- and grafted-NP systems. To this end, we obtain the normal modes for each matrix chain: ${ }^{38,39}$

$$
\mathbf{X}_{\text {matrix }, p}(t)=\frac{1}{L_{\text {matrix }}} \sum_{i=1}^{L_{\text {matrix }}} \mathbf{R}_{\text {matrix }, i}(t) \cos \left(\frac{\pi p(i-1 / 2)}{L_{\text {matrix }}}\right)
$$

where $\mathbf{X}_{\text {matrix }, p}(t)$ are the normal coordinates of the $p$ th mode of a matrix chain at time $t$ and $\mathbf{R}_{\text {matrix }, i}(t)$ is the position vector of the $j$ th bead of the matrix chain. Typically, the relaxation time for each mode is obtained by fitting the time autocorrelation in each mode (relaxation spectrum) $\mathcal{S}_{\text {matrix,p }}(t)$ to a decaying exponential function. However, we found that stretched exponential function provided much better fits to the spectra across different modes:

$$
\begin{aligned}
& \mathcal{S}_{\text {matrix }, p}(t) \equiv \frac{\left\langle\mathbf{X}_{\text {matrix }, p}(t) \cdot \mathbf{X}_{\text {matrix }, p}(0)\right\rangle}{\left\langle\left|\mathbf{X}_{\text {matrix }, p}(0)\right|^{2}\right\rangle} \\
& =\exp \left[-\left(t / \tau_{\text {matrix }, p}\right)^{\beta}\right]
\end{aligned}
$$

where $\tau_{\text {matrix }, p}$ is the relaxation time for the $p$ th mode, $\beta$ is the stretching parameter, and $\langle\cdots\rangle$ denotes ensemble average over all matrix chains and time points.

Figure 3 compares the above relaxation spectra of the five slowest modes $(p=1,2, \ldots, 5)$ of grafted NP systems against those computed for the bare-NP systems. Also included in the figure are the best-fit values of the so-called Rouse relaxation times $\tau_{\text {matrix,R }} \equiv \tau_{\text {matrix,1 }}$ obtained from fitting eq 11 to the spectra of the slowest mode. It can be seen that the computed Rouse times provide good estimates of the frequency at which the storage and loss moduli exhibit an inflection point $(\omega \sim 1 /$ $\left.\tau_{\text {matrix,R }} \approx 10^{-3}\right)$. More importantly, the above results show that the grafting of polymer chains onto the NPs cause only a small $\sim 1-2 \%$ change in the relaxation dynamics of the matrix chains. Moreover, the effect is mixed in the sense that the $d_{\mathrm{NP}}=4 \mathrm{NPs}$ exhibit a slight increase in $\tau_{\text {matrix,R }}$ upon grafting while the two larger NPs exhibit a slight decrease in $\tau_{\text {matrix,R}}$. These marginal and ambivalent changes in the relaxation time of the matrix chains are clearly not responsible for the $\sim 2-7$-fold increase in $G^{\prime}$ and $G^{\prime \prime}$ observed upon grafting.

The above analysis also demonstrates that the matrix chains largely follow Rouse dynamics. In particular, the relaxation spectra decay in a largely exponential manner with the stretching exponent close to unity for the slowest mode $(\beta \approx$ 0.95 for $p=1)$ and decreasing slightly with increasing mode number $(\beta \approx 0.83$ for $p=5)$. Moreover, the relaxation times $\tau_{\text {matrix }, p}$ plotted in Figure S2a of the Supporting Information decay with the characteristic $p^{-2}$ scaling for small $p$ obtained from Rouse theory. The observed Rouse-like dynamics of our matrix polymer is somewhat expected given that the matrix chains are present in a melt-like density where hydrodynamic

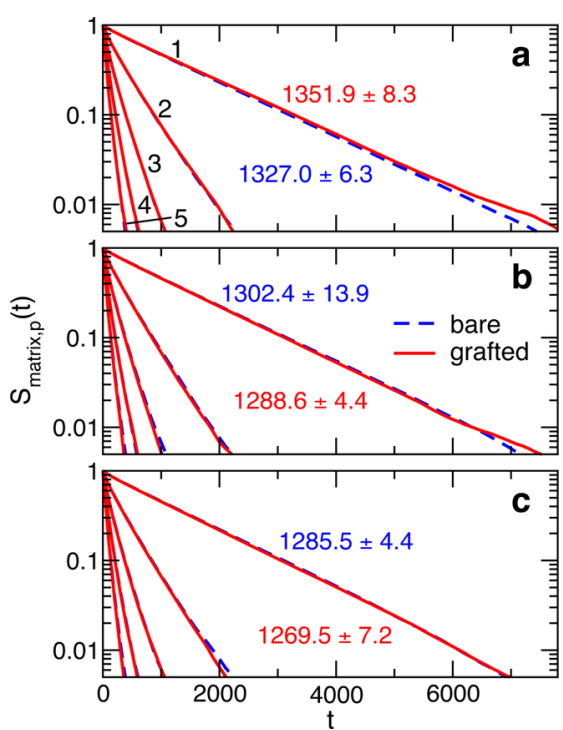

Figure 3. Comparison of the relaxation spectra $\mathcal{S}_{\text {matrix }, p}(t)$ of the matrix chains in PNCs containing bare NPs (blue dashed lines) versus those containing grafted NPs (red solid lines) for the five slowest Rouse modes (labeled 1-5). The spectra are compared for the three NP sizes examined: $d_{\mathrm{NP}}=4(\mathrm{a}), d_{\mathrm{NP}}=6(\mathrm{~b})$, and $d_{\mathrm{NP}}=8(\mathrm{c})$. Also shown in each plot are the relaxation times $\tau_{\text {matrix, } \mathrm{R}}$ associated with the slowest mode for the bare (blue) and grafted NP (red) systems obtained from fitting the spectra to stretched exponentials.

interactions are largely screened out and that the chains are not long enough to display strong entanglements.

To test whether the enhancement in the viscoelastic moduli arose from the slow dynamics of the NP grafts themselves, we compute the Rouse relaxation time of the grafted chains. The normal modes for end-tethered chains are defined slightly differently from those of free chains: ${ }^{40,41}$

$$
\begin{aligned}
& \mathbf{X}_{\text {graft }, p}(t)=\frac{1}{L_{\text {graft }}+1 / 2} \sum_{i=1}^{L_{\text {graft }}}\left(\mathbf{R}_{\text {graft }, i}(t)-\mathbf{R}_{\text {graft }, 0}(t)\right) \\
& \times \sin \left(\frac{\pi i(p-1 / 2)}{L_{\text {graft }}+1 / 2}\right)
\end{aligned}
$$

where $\mathbf{X}_{\text {graft, } p}(t)$ and $\mathbf{R}_{\text {graft, } i}(t)$ denote the normal coordinates and positions of beads on the grafted chain, and $\mathbf{R}_{\text {graft, } 0}(t)$ is the attachment position of the chain on the NP. Figure 4 shows the relaxation spectra $\mathcal{S}_{\text {graft, } p}(t)$ obtained from a time correlation of $\mathbf{X}_{\text {graft, } p}(t)$ corresponding to the five slowest modes of the grafted-NP systems examined above. To obtain the relaxation time, we attempted to fit the relaxation spectra in each mode to a stretched exponential function. However, this did not always provide a good fit, as the spectra seem to be composed of two exponential-like functions. Consequently, we obtain the relaxation times by using the following composite function proposed earlier $^{19}$ that fit our data quite well:

$$
\begin{aligned}
& \mathcal{S}_{\text {graft }, p}(t) \equiv \frac{\left\langle\mathbf{X}_{\text {graft }, p}(t) \cdot \mathbf{X}_{\text {graft }, p}(0)\right\rangle}{\left\langle\left|\mathbf{X}_{\text {graft }, p}(0)\right|^{2}\right\rangle}=\alpha \exp \left[-\left(t / \tau_{\text {graft }, p}\right)^{\beta}\right] \\
& \quad+(1-\alpha) \exp \left(-t / \tau^{\prime}\right)
\end{aligned}
$$

where $\tau_{\text {graft, } p}$ is the relaxation time of the $p$ th mode, $\beta$ is the stretching exponent, and $\alpha$ and $\tau^{\prime}$ represent additional fit parameters. 


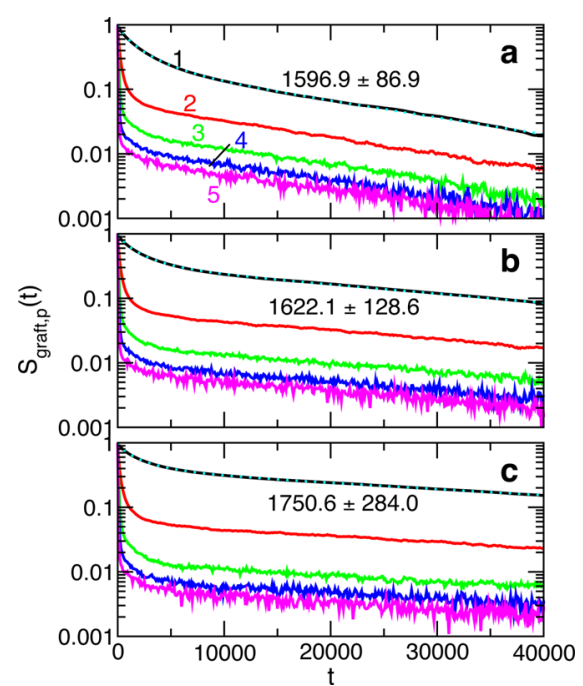

Figure 4. Relaxation spectra $\mathcal{S}_{\text {graft, } p}(t)$ of the five slowest Rouse modes of NP grafts for the three NP sizes examined: $d_{\mathrm{NP}}=4(\mathrm{a}), d_{\mathrm{NP}}=6(\mathrm{~b})$, and $d_{\mathrm{NP}}=8(\mathrm{c})$. Also included in each plot are the Rouse times $\tau_{\text {graft, } \mathrm{R}}$ extracted from fitting the relaxation spectra of the slowest $(p=1)$ mode. The relaxation spectra are shown as solid lines of distinct color for each mode and the fits to the $p=1$ spectra by using eq 13 are shown as dashed cyan lines.

The slowest relaxation times, the Rouse times $\tau_{\text {graft, } R}$ extracted from such fits are provided in Figure 4. We find that the Rouse times are slightly longer than those of the matrix chains, by a factor of $\sim 1.35-1.90$ for the three NP sizes. As discussed later in more detail, Rouse theory ${ }^{39}$ predicts that the storage and loss moduli increase at most quadratically and linearly with the Rouse time, respectively, at low frequencies and even less strongly at high frequencies. Accordingly, the enhancement in the two moduli of the grafted chains should be approximately $1.35-1.9$ and $1.8-3.6$, respectively. The net increase in the overall modulus of the system should be even less, as the grafts constitute only a fraction of the entire system. Thus, the slow relaxation dynamics of the grafts do contribute to the observed enhancement in the moduli but only partially. Moreover, this contribution should be minimal at high frequencies, where stress relaxation and the resultant moduli are dominated by the faster modes that are expected to be similar for graft and matrix chains.

The finding that the grafts exhibit similar or longer relaxation times as the matrix chains seems surprising at the outset given that the grafts $\left(L_{\text {graft }}=20\right)$ are half as long as the matrix chains $\left(L_{\text {matrix }}=40\right)$ and are thereby expected to have roughly 4 times shorter relaxation times as per the Rouse model $\left(\tau_{\text {matrix,R }} \sim\right.$ $L_{\text {matrix }}{ }^{2}$ and $\tau_{\text {graft, } \mathrm{R}} \sim L_{\text {graft }}{ }^{2}$ ). However, it should be noted that the ends of the grafts are fixed to the NPs. The Rouse model predicts that such tethering of one of the ends of a polymer chain should lengthen its relaxation time by a factor of 4 as compared to its free chain counterpart of equivalent length. The two effects should then cancel out to yield roughly similar relaxation times for grafted and matrix chains. That the relaxation times of grafts are even longer than those of the matrix chains might be due to the presence of the NP surface close to the grafts that further slows down their dynamics.

Similar to matrix chains, the grafted chains also seem to follow Rouse dynamics, as noted by the decay in $\tau_{\text {graft, } p}$ with the characteristic $(2 p-1)^{-2}$ scaling obtained from Rouse theory for small $p$ for chains with a fixed end (Figure S2b of
Supporting Information). However, the relaxation spectra are strongly stretched with the exponents ranging from $\beta \approx 0.76$ for $p=1$ to $\beta \approx 0.56$ for $p=3$, suggesting heterogeneous dynamics. Moreover, the spectra indicate the presence of an additional relaxation mechanism, described well by the second term in eq 13 , that is responsible for the slow exponential decay of the tail region of the spectra. This slow decay at large times is likely a signature of the slow rotational motion of the NPs. The reason is that the Rouse modes were calculated using normal coordinates (eq 12) derived for a Gaussian bead-chain fixed at one end to a point and free at the other end, as the normal modes of a chain tethered to an impenetrable surface are not available. The impenetrable NP core prevents the grafted chains from reversing their direction, thereby preventing the asdefined normal modes from completely relaxing. However, the rotational diffusion of the NPs eventually allows the chains to reverse their direction and the modes to relax to zero. We therefore believe that the relatively fast decay of the modes at short times likely arise from the relaxation of the true Rouse modes of the grafted chains while the slower decay likely occurs due to the much slower rotational relaxation of the NPs as discussed earlier. That the time constant $\tau^{\prime}$ for this latter relaxation process remains constant for all modes and that it increases with the size of the NPs, which causes a decrease in their rotational diffusivity, provide further support for the above reasoning.

The above analysis shows that the enhancement in the viscoelastic moduli with NP grafting at low frequencies can be partly explained by the slower relaxation dynamics of the NP grafts compared to matrix chains and, to a smaller extent, by the slight reduction in the relaxation times of the matrix chains. The remainder of the enhancement at low frequencies and the entire enhancement at high frequencies, i.e., the frequencyindependent portion of enhancement going beyond changes in the dynamics of the graft and matrix polymer chains, must then occur due to other effects. We propose that the "strain distortion" effect related to perturbation in the stress and strain fields set up within a fluid or solid due to the presence of hard particles ${ }^{17,20,21}$ is likely responsible for this remaining enhancement due to several reasons. First, it is well-known that this effect enhances the overall viscosity or stiffness of fluids and solids upon particle inclusion independent of changes in intrinsic properties of the host fluid or solid. Second, this effect is expected to lead to a uniform increase in material properties at all frequencies as long as the boundary conditions at the surface of the NPs, typically no-slip boundary conditions, remain independent of frequency. Third, it is well-known that shear distortion, and the resultant enhancement in properties, increases with the number density of particles, which is also consistent with our results in the sense that the grafted NPs, as a result of their grafts, occupy higher volume fractions than bare NPs. While some researchers ${ }^{17}$ have proposed that percolating networks of NPs could also lead to moduli enhancement, these effects tend to arise at much higher particle volume fractions than those examined here.

We next examine whether the observed enhancement in viscoelastic moduli can be quantitatively explained by existing models of viscosity or stiffness enhancement resulting from shear distortion. To this end, we consider three different models proposed by Einstein, ${ }^{21,42}$ Eilers, ${ }^{43,44}$ and Guth ${ }^{20}$ that are commonly used for estimating the shear moduli of composites: 


$$
G_{\mathrm{c}} / G_{0}= \begin{cases}1+2.5 \phi_{\mathrm{p}} & \text { Einstein } \\ 1+2.5 \phi_{\mathrm{p}}+14.1 \phi_{\mathrm{p}}^{2} & \text { Guth } \\ {\left[1+1.25 \phi_{\mathrm{p}} /\left(1-1.35 \phi_{\mathrm{p}}\right)\right]^{2}} & \text { Eilers }\end{cases}
$$

where $\phi_{\mathrm{p}}$ is the volume fraction of hard particles in the composite and $G_{0}$ and $G_{\mathrm{c}}$ are the shear moduli of the pure polymer and the composite, respectively. Einstein derived his model for small $\phi_{\mathrm{p}}$ where the net enhancement in the shear modulus is given by a linear superposition of the shear distortions arising from individual particles; though this relationship was originally derived for shear viscosity of particle suspensions, it is also applicable to a host of other properties, including the shear modulus of composites. Guth extended this model to higher $\phi_{\mathrm{p}}$ by accounting for additional shear distortion arising from the interactions between the distortions arising from neighboring particles. Eilers made empirical corrections to Einstein's model to account for the dramatic rise in the viscosity of suspensions observed when $\phi_{\mathrm{p}}$ approaches close-packing densities.

To compare our simulation results to these models, we calculate the ratios $G^{\prime} / G_{0}^{\prime}$ and $G^{\prime \prime} / G_{0}^{\prime \prime}$ of the moduli obtained for our bare and grafted NP systems to those of our pure polymer system denoted by $G_{0}^{\prime}$ and $G_{0}^{\prime \prime}$ (provided in Figure S3 of the Supporting Information). While the particle volume fraction $\phi_{\mathrm{p}}$ in eq 14 is simply equal to $\phi_{\mathrm{NP}}$ for bare NPs, the above models do not specify the definition of $\phi_{\mathrm{p}}$ for grafted NPs. As a reasonable approximation, we consider it as the sum of the volume fractions of the NPs $\phi_{\mathrm{NP}}$ and that of the grafts $\phi_{\text {graft: }}$

$$
\phi_{\mathrm{p}}=\phi_{\mathrm{NP}}+\phi_{\text {graft }}=\phi_{\mathrm{NP}}+n_{\mathrm{NP}} \Gamma_{\text {graft }} L_{\text {graft }} v_{0} / V
$$

where $V$ is the volume of the simulation box and $v_{0} \equiv 1 / \rho_{\text {polymer }}$ is the effective volume occupied by each polymer bead. The three bare NP systems of different sizes all exhibit the same $\phi_{\mathrm{p}}$ $=0.027$. However, the grafted NP systems exhibit variable $\phi_{\mathrm{p}}$ for the three NP sizes, increasing from 0.221 to 0.416 as $d_{\mathrm{NP}}$ decreases from 8 to 4 ; this increase in $\phi_{\mathrm{p}}$ occurs due to an increase in the net surface area of the NPs as they become smaller that leads to a higher number of total grafted chains in the system (given that the grafting density remains constant across the three NP sizes). Furthermore, we calculated the above ratios for low- and high-frequency regimes by averaging the moduli ratios over $\omega \in[0.00001-0.0005]$ and $\omega \in[0.1-$ $1]$, respectively. Since grafting-induced changes in relaxation time of polymer chains are expected to affect only the lowfrequency moduli, comparing the moduli ratios across the two frequency regimes may allow us to extricate the contribution of strain distortion from that due to the slowdown in the dynamics of the chains.

Figures $5 \mathrm{a}$ and $5 \mathrm{~b}$ show the computed $G^{\prime} / G_{0}^{\prime}$ and $G^{\prime \prime} / G_{0}^{\prime \prime}$ ratios plotted against $\phi_{\mathrm{p}}$ for the six PNCs containing bare and grafted NPs at low- and high-frequency regimes along with the $G_{c} / G_{0}$ predicted from eq 14 . Our results show that both $G^{\prime} / G_{0}^{\prime}$ and $G^{\prime \prime} / G_{0}^{\prime \prime}$ increase with increasing $\phi_{\mathrm{p}}$, consistent with the trend obtained from the strain distortion models, though the different models differ somewhat from each other. Moreover, the moduli ratio computed from simulations at high frequencies are of comparable magnitude to those predicted by the models, especially the model proposed by Guth, whereas the ratios computed at low frequencies tend to exceed all model
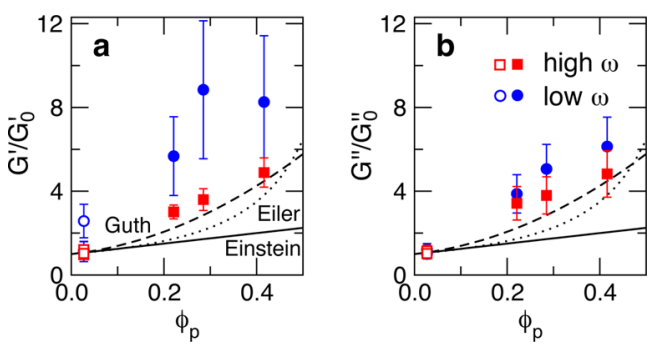

Figure 5. Ratio of storage (a) and loss modulus (b) of the three bare$\mathrm{NP}$ and the three grafted-NP systems to that of pure polymer plotted as a function of the effective NP volume fraction. The moduli ratios obtained from simulations at low and high frequencies are shown as blue circles and red squares, respectively. Open symbols represent bare NPs, and filled symbols represent grafted NPs. The black solid, dotted, and dashed lines represent predictions from the models of Einstein, Eilers, and Guth, respectively.

predictions. This is consistent with our hypothesis that the enhancement in moduli at low frequencies arises from a combination of strain distortion and slowdown in chain dynamics while that at high frequencies arises from strain distortion effects alone. We also note that at low frequencies the computed $G^{\prime} / G_{0}^{\prime}$ ratios more strongly exceed the model predictions as compared to $G^{\prime \prime} / G_{0}^{\prime \prime}$, which is also consistent with the expectation that $G^{\prime}$ is more strongly affected by changes in the relaxation times of the polymer chains (quadratic dependence with the Rouse time) as compared to $G^{\prime \prime}$ (linear dependence). The above results provide further support for the strain distortion effect as being responsible for the frequency-independent portion of the enhancement in viscoelastic moduli observed due to NP grafting. Our results also show that considering the effective volume fraction of the particulate phase in eq 14 in the case of grafted NPs as the sum of the volume fractions of the hard NP cores and of the attached NP grafts may be a reasonable approximation.

In summary, our simulation results show that grafting NPs with polymer chains, even those that interact neutrally with the polymer matrix, results in a substantial enhancement in $G^{\prime}$ and $G^{\prime \prime}$ across the entire frequency range, with the enhancement being slightly larger at lower frequencies. Our analyses suggest that the frequency-independent portion of this enhancement arises from a known hydrodynamic effect whereby embedding of particles within a polymer distorts the shear field in the polymer, leading to enhanced stiffness and dissipation, while the frequency-dependent portion arises from slower relaxation dynamics of the grafted chains as compared to the matrix chains, despite their shorter length, leading to additional moduli enhancement at low frequencies. It is interesting to note that another study ${ }^{45}$ performed simulations of PNCs below their $T_{\mathrm{g}}$ to compare the effects of bare versus grafted NPs on the elastic constants and postyield properties of PNCs and obtained results that are somewhat different from our study here, which examines PNCs at temperatures well above $T_{\mathrm{g}}$. It was found that properties such as elastic constants, which are not significantly affected by segmental mobility below $T_{\mathrm{g}}$, were largely unaffected by NP grafting, while properties like strain hardening, where segmental mobility now becomes important, were found to be more pronounced in systems with grafted particles.

Effect of Graft/Matrix Interactions. It was previously shown for bare NPs that increasing attraction between the NPs and the polymer matrix led to a significant slowdown in the 
relaxation time of the polymer chains in close proximity to the NPs, resulting in a large increase in the viscosity. ${ }^{19}$ To investigate if a similar effect occurs in the case of grafted chains on NPs, we introduce mutual attraction between $n_{\text {att }}$ terminal beads of the grafted and $n_{\text {att }}$ terminal and centrally located beads of the matrix chains mimicking strong intermolecular interactions between spaced functional groups on polymers. To study the effect of increasing attraction, we vary the number of such attractively interacting beads from 0 to 2 , thereby generating three systems with different extents of attraction, which we refer to as neutral (the original grafted-NP system studied above with $\left.n_{\text {att }}=0\right)$, weakly attractive $\left(n_{\text {att }}=1\right)$, and strongly attractive $\left(n_{\mathrm{att}}=2\right)$.

Figure 6 shows that increasing attraction between the grafted and matrix chains leads to an increase in $G^{\prime}(\omega)$ and $G^{\prime \prime}(\omega)$.
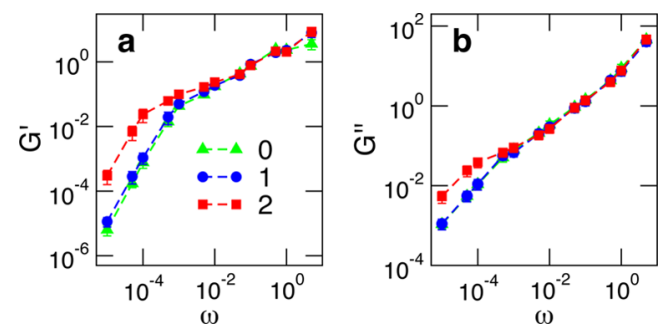

Figure 6. Comparison of the storage (a) and loss modulus (b) of polymers containing grafted NPs for three different strengths of attraction between the grafted and matrix chains: $n_{\text {att }}=0$ (green triangles), $n_{\text {att }}=1$ (blue circles), and $n_{\text {att }}=2$ (red squares). The symbols represent computed data while dashed lines are guides to the eye.

Unlike the effects of grafting observed earlier, the enhancement in the moduli observed here is highly frequency dependent with strong enhancement at low frequencies and negligible enhancement at high frequencies. The two regimes are clearly demarcated by the kink in the moduli at a frequency associated with the characteristic relaxation time of the polymer. This sharp frequency dependence already gives us a cue that shear distortion is unlikely to be the underlying mechanism for the observed enhancement and that it is more likely due to fundamental changes in polymer dynamics. Furthermore, the effect of increasing attraction is highly nonlinear in the sense that the introduction of one pair of mutually attractive matrix and graft beads $\left(n_{\text {att }}=1\right)$ causes only a small increase in $G^{\prime}$ and $G^{\prime \prime}$, but the introduction of another pair of attractive beads $\left(n_{\text {att }}\right.$ $=2$ ) leads to a dramatic enhancement. In fact, the fold enhancement in the moduli of attractive grafts over those of neutral grafts approaches values as large as $47 \pm 28$ in $G^{\prime}$ and 5 \pm 2 in $G^{\prime \prime}$ for strongly attractive grafts at the lowest frequency investigated here $\left(\omega=10^{-5}\right)$. These results suggest that raising the affinity between NP grafts and the matrix polymer could be a powerful approach for enhancing the viscoelastic properties of PNCs.

To confirm that the observed enhancement in the moduli arises from a slowdown in the dynamics of the polymer chains, we calculate the Rouse relaxation times of the matrix chains and of the grafted chains using the methods outlined earlier. Figure 7 shows the relaxation spectra for the five slowest Rouse modes of the matrix chains for neutral, weakly attractive, and strongly attractive systems, and Figure 8 shows the corresponding spectra for the grafted chains. The relaxation spectra of the matrix chains in the weakly attractive system (Figure 7b)

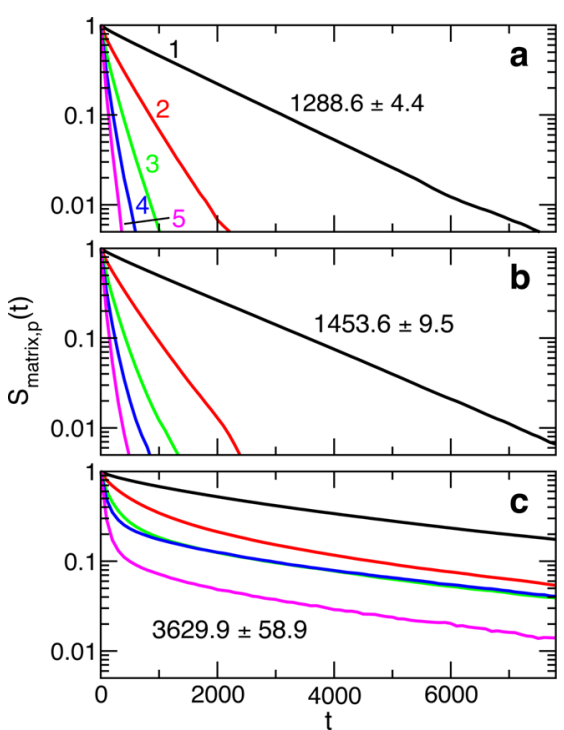

Figure 7. Relaxation spectra $\mathcal{S}_{\text {matrix }, p}(t)$ of the five slowest Rouse modes of the matrix chains for PNCs containing NPs grafted with neutral chains (a), weakly attractive chains (b), and strongly attractive chains (c). Also included in each plot are the relaxation times $\tau_{\text {matrix,R }}$ associated with the slowest mode $(p=1)$ for the three systems obtained from fitting the spectra to stretched exponentials.

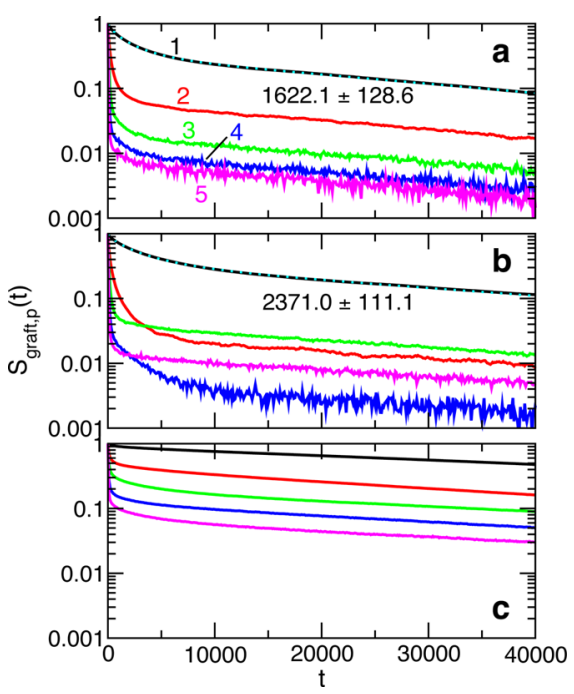

Figure 8. Relaxation spectra $\mathcal{S}_{\text {graft, }}(t)$ of the five slowest Rouse modes of the grafted chains for PNCs containing NPs grafted with neutral chains (a), weakly attractive chains (b), and strongly attractive chains (c). Also included in each plot are the relaxation times $\tau_{\text {graft, } R}$ associated with the slowest mode $(p=1)$ for neutral and weakly attractive grafts. The spectra for strongly attractive grafts could not be fitted to any combination of exponential or stretched exponential functions.

exhibit near-exponential decay, similar to that observed in the neutral system (Figure 7a) albeit with longer relaxation times. Specifically, the Rouse time in the weakly attractive system is $\tau_{\text {matrix, } \mathrm{R}} \approx 1454$, about $13 \%$ higher than the corresponding $\tau_{\text {matrix } \mathrm{R}}$ for the neutral system. We also note that the relaxation spectra remains Rouse-like with the stretching exponent ranging from $\beta=0.93$ to $\beta=0.78$ for $p=1$ to $p=5$. The relaxation spectra of the grafted chains in the weakly attractive systems (Figure $8 \mathrm{~b}$ ), in contrast, exhibit a highly stretched 
exponential decay similar to neutral systems (Figure 8a). More importantly, the grafted chains in this weakly attractive system exhibited a Rouse time of $\tau_{\text {graft, } R} \approx 2371$, about $46 \%$ higher than the corresponding $\tau_{\text {graft, } R}$ for the neutral system. Thus, the moderate slowdown in the relaxation time of the matrix and grafted chains with increased attraction could indeed be responsible for the moderate enhancements in $G^{\prime}$ and $G^{\prime \prime}$ observed at low frequencies.

Turning to the strongly attractive system, we note striking differences in the relaxation spectra of matrix chains here (Figure 7c) compared to the neutral and weakly attractive systems. In particular, the spectra exhibit "non-Rouse" behavior, with a small stretching exponent of $\beta=0.72$ for $p=1$ that further drops down to $\beta=0.26$ for $p=5$. Such heavy stretching of the spectra indicates heterogeneous dynamics of the chains. Indeed, the matrix chains could be present in multiple possible states-chains bound to a grafted chain through the middle, end, or both pairs of attractive beads, chains bound to multiple grafted chains via the two pairs of attractive beads, and chains not bound to any grafted chains-resulting in a spectrum of distinct relaxation times. By fitting the curves to stretched exponentials, we obtained a significantly longer relaxation time of $\tau_{\text {matrix, } \mathrm{R}} \approx 3630$, nearly 3 times the relaxation time of neutral chains. According to Rouse scalings, the low-frequency storage and loss modulus should be $\sim 9$ and $\sim 3$ times larger. The relaxation spectrum of the grafted chains for the strongly attractive system (Figure $8 \mathrm{c}$ ) are also heavily stretched such that they can no longer be fitted via eq 13, making it difficult to obtain the corresponding relaxation times. Nevertheless, it is evident that the relaxation spectra decay much slower than those of the neutral and weakly attractive systems. The above results clearly indicate that the dramatic enhancement in $G^{\prime}$ and $G^{\prime \prime}$ observed for strongly attractive grafts could result from the dramatic slowdown in the dynamics of both the grafted and matrix chains.

We also note nonmonotonic behavior in the shapes of the mode spectra for the attractive systems, most notably in Figure $8 \mathrm{c}$ where the spectra of modes $p=2$ and $p=3$ and then $p=4$ and $p=5$ are seen to intersect. The origin of this peculiar behavior is unknown, but it could occur due to a combination of glassy dynamics in the strongly attractive system ${ }^{46}$ (as noted from the slow rotational relaxation times of the NPs) and periodic attraction between the grafted chains and matrix chains. Since each Rouse mode probes the dynamics of different portions of the chains, each mode is affected differently by the attractive restraints. In other words, modes that depend strongly on the mobility of the attractive beads are affected more than those modes that do not depend so strongly. Note that the transformations we employ for converting Cartesian coordinates to normal coordinates for the matrix (eq 10) and grafted chains (eq 12) do not account for these restraints.

It is also intriguing that the difference in the effects of one and two attractive beads is so dramatic. This effect most likely arises from cooperativity in the binding of matrix chains to the grafts, similar to the phenomenon common in biological systems, where interacting biomolecules with two binding sites bind much more favorably than those with a single binding site. That is, the free energy of binding of a matrix chain to a grafted chain via two adjacent attractive beads versus one attractive bead is much larger than 2-fold, as the former binding event costs only slightly larger translation, rotational, and configurational entropy but gains twice the favorable binding enthalpy, leading to significantly larger overall binding free energy. We provide a rough calculation involving radial distribution functions in the Supporting Information (Figure S4) for estimating the difference in the binding free energy, and its enthalpic and entropic components, between the two kinds of interactions, i.e., between single attractive beads and between dimers of attractive beads embedded within the grafted and matrix chains.

Effects of Other NP-Associated Parameters. We next examine how other parameters of the grafted NPs-their loading, size, grafted chain length, and grafting density-affect the viscoelastic moduli of the PNCs. We demonstrated earlier that these properties of PNCs are governed by two main mechanisms: distortion of the shear field caused by NPs leading to changes in the stiffness and dissipation of the PNCs and changes in the dynamics of the matrix and grafted chains directly affecting stress relaxation in the PNC. We found that these two effects could be reasonably characterized in terms of the effective particle volume fraction $\phi_{\mathrm{p}}$ (inclusive of grafts) and the relaxation times $\tau_{\text {matrix,R }}$ and $\tau_{\text {graft, } \mathrm{R}}$ of the matrix and grafted chains. Below we investigate the effects of the NP-associated parameters mentioned above on the $G^{\prime}(\omega)$ and $G^{\prime \prime}(\omega)$ of PNCs and attempt to understand the effects in light of these two mechanisms of moduli enhancement, i.e., in terms of $\phi_{\mathrm{p}}$, $\tau_{\text {matrix,R }}$ and $\tau_{\text {graft,R }}$ obtained for each PNC system studied here (Table 2).

We begin with the effects of NP loading. Figure 9a,b plots the $G^{\prime}(\omega)$ and $G^{\prime \prime}(\omega)$ profiles for $d_{\mathrm{NP}}=6$ grafted NPs at two different loadings of 6 and $12 \mathrm{wt} \%$. The corresponding profiles for NPs with $d_{\mathrm{NP}}=4$ and 8 are plotted in Figure S5 of the Supporting Information. All three sets of results show a considerable increase in $G^{\prime}(\omega)$ and $G^{\prime \prime}(\omega)$ with an increase in NP loading, in good agreement with previous $\mathrm{MD}$ simulations of bare NP-polymer systems ${ }^{17}$ and experimental measurements on bare and grafted NP composites ${ }^{16,14}$ showing similar enhancement with increasing loading. We note some frequency dependence in the above enhancement, stronger than that observed when examining the effects of grafting of NPs (Figure 2) but much weaker than that observed when examining the effects of increasing attraction between grafted and matrix chains (Figure 6). According to our previous analyses, we attribute the frequency-independent portion of this enhancement to shear distortion, whereby an increase in the number of NPs within a given volume of the PNC is expected to increase the amount of distortion in the shear and stress fields caused by the NPs. The remaining frequency-dependent portion of the enhancement, i.e., the enhancement observed at low frequencies, is attributed to a slowdown in the relaxation time of both the matrix and grafted chains, as confirmed by the increase in the corresponding $\tau_{\text {matrix,R }}$ and $\tau_{\text {graft, } R}$ with NP loading (Table 2), especially for systems containing the smallest NPs. The increase in $\tau_{\text {matrix,R }}$ likely occurs due to a decrease in the average spacing between the NPs with increase NP loading, which leads to increased confinement of the matrix chains and increase in their interactions with the less mobile grafts. The increase in $\tau_{\text {graft, } R}$ likely occurs due to increased interpenetration between the grafts on neighboring NPs. In other words, as the NP loading increases, a larger fraction of grafts originally interacting with the mobile matrix chains now interact with the less mobile grafts on neighboring NPs, leading to slower dynamics of the grafted chains. A more detailed analysis of the effects of grafted and matrix chains on the dynamics of NPs is provided in refs 47 and 48. 
Table 2. Effective Volume Fraction of Grafted NPs and Rouse Relaxation Times of Polymer Matrix and Grafted Chains

\begin{tabular}{|c|c|c|c|c|}
\hline $\begin{array}{l}\text { system } \\
\text { no. }{ }^{a}\end{array}$ & $\begin{array}{l}\text { parameter } \\
\text { change }^{b}\end{array}$ & $\begin{array}{c}\text { vol frac } \\
\phi_{\mathrm{p}}\end{array}$ & $\begin{array}{c}\text { Rouse time } \\
\tau_{\text {matrix }, \mathrm{R}}\end{array}$ & $\begin{array}{c}\text { Rouse time } \\
\tau_{\text {graft, } \mathrm{R}}\end{array}$ \\
\hline & $\mathrm{B} / \mathrm{G}$ & & NP grafting & \\
\hline 1 & B & 0.027 & $1327 \pm 6$ & NA \\
\hline 2 & G & 0.416 & $1352 \pm 8$ & $1597 \pm 87$ \\
\hline 3 & B & 0.027 & $1302 \pm 14$ & NA \\
\hline 4 & G & 0.286 & $1289 \pm 4$ & $1622 \pm 129$ \\
\hline 5 & B & 0.027 & $1285 \pm 4$ & NA \\
\hline 6 & $\begin{array}{l}\mathrm{G} \\
d_{\mathrm{NP}}\end{array}$ & 0.221 & $\begin{array}{l}1269 \pm 7 \\
\text { NP size }\end{array}$ & $1751 \pm 284$ \\
\hline 2 & 4.0 & 0.416 & $1352 \pm 8$ & $1597 \pm 87$ \\
\hline 4 & 6.0 & 0.286 & $1289 \pm 4$ & $1622 \pm 129$ \\
\hline 6 & $\begin{array}{l}8.0 \\
f_{\mathrm{NP}}\end{array}$ & 0.221 & $\begin{array}{l}1269 \pm 7 \\
\text { NP loading }\end{array}$ & $1751 \pm 284$ \\
\hline 2 & 0.06 & 0.416 & $1352 \pm 8$ & $1597 \pm 87$ \\
\hline 7 & 0.12 & 0.847 & $1553 \pm 23$ & $1775 \pm 73$ \\
\hline 4 & 0.06 & 0.286 & $1289 \pm 4$ & $1622 \pm 129$ \\
\hline 8 & 0.12 & 0.592 & $1364 \pm 11$ & $1800 \pm 77$ \\
\hline 6 & 0.06 & 0.221 & $1269 \pm 7$ & $1751 \pm 284$ \\
\hline 9 & $\begin{array}{l}0.12 \\
L_{\text {graft }}\end{array}$ & 0.458 & $\begin{array}{l}1312 \pm 10 \\
\text { graft length }\end{array}$ & $1661 \pm 84$ \\
\hline 10 & 10 & 0.156 & $1288 \pm 5$ & $395 \pm 13$ \\
\hline 4 & 20 & 0.286 & $1289 \pm 4$ & $1622 \pm 129$ \\
\hline 11 & 30 & 0.415 & $1312 \pm 10$ & $3594 \pm 161$ \\
\hline 12 & $\begin{array}{l}40 \\
\Gamma_{\text {graft }}\end{array}$ & 0.544 & $\begin{array}{l}1333 \pm 12 \\
\text { graft density }\end{array}$ & $5849 \pm 953$ \\
\hline 13 & 0.1 & 0.090 & $1298 \pm 2$ & $1347 \pm 127$ \\
\hline 14 & 0.2 & 0.102 & $1298 \pm 6$ & $1593 \pm 26$ \\
\hline 4 & 0.4 & 0.286 & $1289 \pm 4$ & $1622 \pm 129$ \\
\hline 15 & $\begin{array}{l}0.8 \\
n_{\text {att }}\end{array}$ & 0.544 & $\begin{array}{l}1279 \pm 5 \\
\text { graft/matrix } \\
\text { affinity }\end{array}$ & $1576 \pm 111$ \\
\hline 2 & 0 & 0.416 & $1352 \pm 8$ & $1597 \pm 87$ \\
\hline 16 & 1 & 0.027 & $1453 \pm 10$ & $2371 \pm 111$ \\
\hline 17 & 2 & 0.027 & $3630 \pm 59$ & no fit \\
\hline
\end{tabular}

${ }^{a}$ Index identifying different simulation system (same as Table 1). ${ }^{b}$ Only the parameter being changed in the comparison group of simulation is tabulated (refer to Table 1 for the entire list of fixed parameters).

Next, we examined the effects of NP size. Figure 9c,d compares the $G^{\prime}(\omega)$ and $G^{\prime \prime}(\omega)$ for grafted NPs of three different sizes: $d_{\mathrm{NP}}=4,6$, and 8 . We observe a monotonic decrease in $G^{\prime}(\omega)$ and $G^{\prime \prime}(\omega)$ with increasing NP size, suggesting that smaller NPs provide more reinforcement than larger ones. An analogous effect has been observed experimentally, ${ }^{49-51}$ where the Young's modulus of various PNCs was observed to decrease with increasing NP size at fixed NP wt \%, with the decrease becoming more pronounced for high NP loadings and small NP sizes. We also note that the computed decrease in the two moduli is largely frequency independent; this is more evident in the $G^{\prime \prime}$ profiles that carry less statistical uncertainties than the $G^{\prime}$ profiles. Thus, the observed effect of NP size is likely due to shear-distortion effects and not due to intrinsic changes in the relaxation time of the matrix polymer. Computations of the effective volume fraction of the grafted NPs and the relaxation times of the matrix and grafted chains showing a large decrease in $\phi_{\mathrm{p}}$ and a minor decrease in $\tau_{\text {matrix,R }}$ and statistically insignificant change in $\tau_{\text {graft, } \mathrm{R}}$ with increasing NP size confirms the above notion.
The decrease in $\phi_{\mathrm{p}}$ with $d_{\mathrm{NP}}$ can be understood by noting that as the NPs become larger, their number density within the PNC needs to decrease to keep the net NP loading $f_{\mathrm{NP}}$ fixed. This naturally leads to a reduction in the net surface area of the NPs and a concomitant decrease in the total number of grafted chains $n_{\text {graft }}$ causing the effective volume fraction of the NPs to decrease. The minor decrease in $\tau_{\text {matrix,R }}$ also likely results from the decrease in $\phi_{\mathrm{p}}$ that alleviates the confinement experienced by the matrix chains, causing a slight speed-up in their dynamics.

Figure 9e,f plots the effects of graft length on the computed $G^{\prime}$ and $G^{\prime \prime}$ for NPs grafted with chains of lengths $L_{\text {graft }}=10-40$. We observe a clear enhancement in $G^{\prime}(\omega)$ and $G^{\prime \prime}(\omega)$ with increasing $L_{\text {graft }}$ at low frequencies but virtually no enhancement at high frequencies. This strong frequency dependence immediately suggests that the observed moduli enhancement are likely due to changes in the dynamics of the chains. Indeed, the Rouse times $\tau_{\text {graft,R }}$ of the grafted chains increase drastically with increasing chain length (Table 2 ), from $\tau_{\text {graft, } \mathrm{R}} \approx 395$ for $L_{\text {graft }}=10$ to $\tau_{\text {graft, } \mathrm{R}} \approx 5949$ for $L_{\text {graft }}=40$, consistent with the predictions of Rouse theory: $\tau_{\text {graft, } \mathrm{R}} \sim L_{\text {graft }}{ }^{2}$. Interestingly, the relaxation time of the matrix chains also rises with increasing $L_{\text {graft }}$ though this increase is much smaller ( $\sim 20 \%$ rise in $\tau_{\text {matrix,R }}$ in going from the shortest to the longest graft). Thus, the longer the grafts, the more they apparently entangle with the matrix chains, leading to a slowdown in their dynamics. Thus, the drastic increase in the relaxation time of the graft can explain the $\sim 3$ - and $\sim 4$-fold increase in $G^{\prime}$ and $G^{\prime \prime}$ at low frequencies observed in going from $L_{\text {graft }}=10$ to 40 . Surprisingly, even though the longer grafts increase the effective volume fraction $\phi_{\mathrm{p}}$ of the NPs, they do not result in the expected frequency-independent enhancement in the two moduli, as seen with NP loading. A plausible explanation for this result emerges from our analysis of the polymer density around a grafted NP, in terms of number of polymer beads per unit volume, as a function of radial distance $r$ from the NP center. Figure S6 in the Supporting Information plots the three contributions to this polymer density arising from the NP's own grafted chains $\rho_{\text {graft }}(r)$, from grafted chains of neighboring NPs $\rho_{\text {graft-n }}(r)$, and from matrix chains $\rho_{\text {matrix }}(r)$. We observe considerable overlap in the $\rho_{\text {graft }}(r)$ and $\rho_{\text {matrix }}(r)$ profiles, indicating significant penetration of the matrix chains into the grafted chains on the NPs and some overlap between $\rho_{\text {graft }}(r)$ and $\rho_{\text {graft-n }}(r)$ profiles, indicating interpenetration of grafted chains across neighboring NPs. Importantly, for all four graft lengths investigated here, the matrix chains are able to penetrate through the layer of grafted chains all the way to the NP surface. Thus, the shear-distortion effect apparently does not occur due to absence of any depletion of available volume to the matrix chains.

Lastly, we examined the effect of grafting density. Figure 9g,h displays the $G^{\prime}(\omega)$ and $G^{\prime \prime}(\omega)$ profiles computed for NPs grafted at four different grafting densities in the range $\Gamma_{\text {graft }}=$ $0.1-0.8$. The computed $G^{\prime}$ and $G^{\prime \prime}$ increase monotonically with increasing $\Gamma_{\text {graft }}$. As opposed to the effects of increasing graft length, the moduli enhancement in this case is more or less frequency independent; again, the $G^{\prime \prime}$ plots provide clearer trends due to their smaller statistical errors. Thus, shear distortion and not changes in the relaxation time of the chains is likely responsible for the observed enhancement. Indeed, the computed Rouse times $\tau_{\text {matrix, } \mathrm{R}}$ and $\tau_{\text {graft, } \mathrm{R}}$ remain rather insensitive to changes in $\Gamma_{\text {graft }}$ (Table 2 ). To address the question as to why increasing $\Gamma_{\text {graft }}$ leads to more strain 


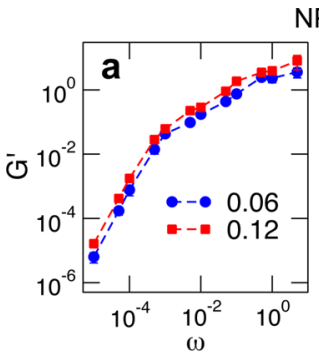

NP loading

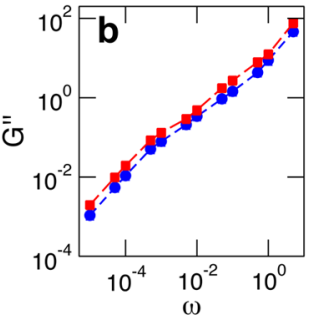

Graft length
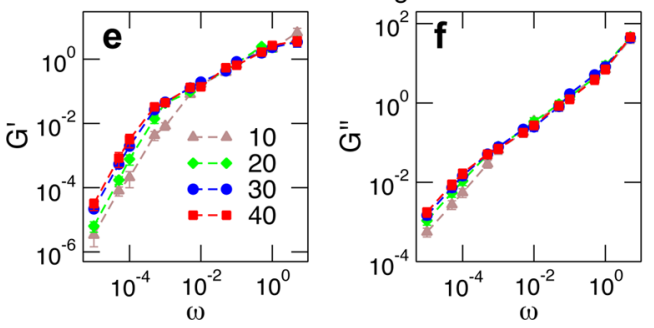

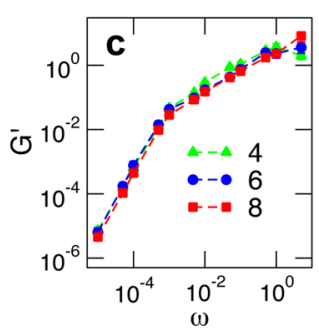

NP size
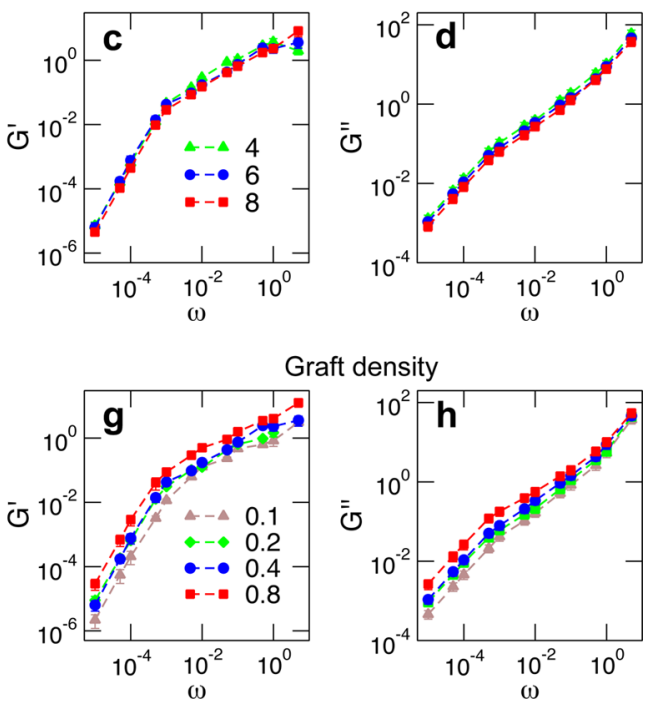

Figure 9. Comparison of the storage and loss modulus of polymers containing grafted NPs at (a) two different loadings of $f_{\mathrm{NP}}=0.06$ (blue circles) and 0.12 (red squares) for size $d_{\mathrm{NP}}=6$; (b) three different sizes of $d_{\mathrm{NP}}=4$ (green triangles), 6 (blue circles), and 8 (red squares); (c) four different graft lengths of $L_{\text {graft }}=10$ (brown triangles), 20 (green diamonds), 30 (blue circles), and 40 (red squares); and (d) four different grafting densities of $\Gamma_{\text {graft }}=0.1$ (brown triangles), 0.2 (green diamonds), 0.4 (blue circles), and 0.8 (red squares). The symbols represent computed data while dashed lines are guides to the eye.

distortion while increasing $L_{\text {graft }}$ led to no such effect, we again computed the polymer density profiles (Figure S6 in Supporting Information). Interestingly, we find that in this case increasing the number of grafted chains does lead to exclusion of matrix chains from the surface of the NPs, consistent with a wetting-to-dewetting transition observed with increasing grafting density observed by others, ${ }^{52}$ causing increased strain distortion. Thus, contrary to intuition, the effects of increasing grafting density and increasing graft lengths are fundamentally quite different.

The above results demonstrate that a rich spectrum of effects could arise from modulating the different NP-related parameters and that it may be possible to modulate the viscoelastic properties of PNCs through a variety of parameters. For instance, if uniform enhancement in the viscoelastic moduli across all frequencies is desired, the grafting density and the NP loading density would be useful parameters to modulate. On the other hand, if the enhancement is desired only at low frequencies, one could modulate the attraction between the grafts and the matrix chains and the length of the grafted chains. The variety of such control parameters implies that targeted viscoelastic properties could be achieved while satisfying other design constraints, e.g., maintaining good dispersion of NPs within the polymer matrix.

Phenomenological Model. We next attempt to build a simple phenomenological model to quantitatively capture the viscoelastic properties of our PNCs, specifically their dependence on the different NP-related parameters examined in this study. Our results so far show that the viscoelastic properties of PNCs are governed by (1) the effective volume fraction of the NPs, which dictates the amount of shear distortion in the PNC and gives rise the frequency-independent enhancement in the viscoelastic moduli, and (2) the relaxation times of the matrix and grafted chains, which dictate stress relaxation in the PNC and give rise to the frequency-dependent enhancement in the moduli. On the basis of these observations, we propose that the storage $G^{\prime}(\omega)$ and loss modulus $G^{\prime \prime}(\omega)$ of the PNC can be described to first approximation by the following composite function:

$$
\begin{aligned}
& G^{\prime}(\omega) \simeq G_{\text {polymer }}^{\prime}(\omega) f_{\text {particle }}\left(\phi_{\mathrm{p}}\right) \\
& G^{\prime \prime}(\omega) \simeq G_{\text {polymer }}^{\prime \prime}(\omega) f_{\text {particle }}\left(\phi_{\mathrm{p}}\right)
\end{aligned}
$$

where $G_{\text {polymer }}^{\prime}(\omega)$ and $G_{\text {polymer }}^{\prime \prime}(\omega)$ represent the frequencydependent moduli of the polymer portion of the PNC accounting for changes in the relaxation dynamics of the grafted and/or matrix chains, while $f_{\text {particle }}\left(\phi_{\mathrm{p}}\right)$ accounts for additional frequency-independent enhancement in the PNC moduli due to shear distortion arising from particle inclusions independent of polymer properties.

A reasonable starting point for estimating $f_{\text {particle }}\left(\phi_{\mathrm{p}}\right)$ is the model proposed by Einstein, ${ }^{21,42}$ where the particle volume fraction is replaced by the effective volume fraction of the grafted NPs inclusive of the grafted chains:

$$
f_{\text {particle }}\left(\phi_{\mathrm{p}}\right) \simeq 1+2.5 \phi_{\mathrm{p}}=1+2.5\left(\phi_{\mathrm{NP}}+\phi_{\text {graft }}\right)
$$

We also considered the more advanced model by Guth, ${ }^{20}$ which accounts for two-body terms when calculating shear distortion, but found that the Einstein model yielded better agreement with the simulated data. It is concievable that these higher-order shear distortion terms might even be suppressed in polymer chain melts given that hydrodynamic interactions are known to be largely screened out in such polymer melts.

To formulate a model for estimating $G_{\text {polymer }}^{\prime}(\omega)$ and $G_{\text {polymer }}^{\prime \prime}(\omega)$, we make the ansatz that these "mixture" moduli are related to the "pure-component" moduli of the individual polymeric constituents of the PNC, i.e., $G_{\text {matrix }}^{\prime}(\omega)$ and $G_{\text {matrix }}^{\prime \prime}(\omega)$ of the matrix chains and $G_{\text {graft }}^{\prime}(\omega)$ and $G_{\text {graft }}^{\prime \prime}(\omega)$ of the grafted chains, which in turn depend on their respective sets of chain relaxation times $\left\{\tau_{\text {matrix }}\right\}$ and $\left\{\tau_{\text {graft }}\right\}$, respectively. The mixture moduli can then be formulated as a function $g$ of the pure-component moduli appropriately weighted by their respective volume fractions $\phi_{\text {matrix }}$ and $\phi_{\text {graft }}$ of the matrix and grafted chains. In other words 


$$
G_{\text {polymer }}^{\prime}(\omega)=g\left(G_{\text {matrix }}^{\prime}\left(\omega ;\left\{\tau_{\text {matrix }}\right\}\right), G_{\text {graft }}^{\prime}\left(\omega ;\left\{\phi_{\text {graft }}\right\}\right), \phi_{\text {matrix }}, \phi_{\text {graft }}\right)
$$

$$
G_{\text {polymer }}^{\prime \prime}(\omega)=g\left(G_{\text {matrix }}^{\prime \prime}\left(\omega ;\left\{\tau_{\text {matrix }}\right\}\right), G_{\text {graft }}^{\prime \prime}\left(\omega ;\left\{\phi_{\text {graft }}\right\}\right), \phi_{\text {matrix }}, \phi_{\text {graft }}\right)
$$

We attempted both resistances-in-parallel and resistances-inseries models ${ }^{53}$ for the function $g$ in order to estimate the mixture moduli from the pure-component moduli but found that the latter model yielded estimates that better agree with our simulation results. Hence, we report only the results from the resistances-in-series model, according to which the mixture moduli is given by

$$
\begin{aligned}
& G_{\text {polymer }}^{\prime}(\omega)=\left[\frac{\phi_{\text {matrix }}}{G_{\text {matrix }}^{\prime}(\omega)}+\frac{\phi_{\text {graft }}}{G_{\text {graft }}^{\prime}(\omega)}\right]^{-1} \\
& G_{\text {polymer }}^{\prime \prime}(\omega)=\left[\frac{\phi_{\text {matrix }}}{G_{\text {matrix }}^{\prime \prime}(\omega)}+\frac{\phi_{\text {graft }}}{G_{\text {graft }}^{\prime \prime}(\omega)}\right]^{-1}
\end{aligned}
$$

To estimate the pure-component moduli, we turn to the Rouse model. The polymer chains in most of our simulated systems follow Rouse dynamics, as evident from the nearexponential decay of the relaxation spectra, albeit with some stretching in the case of the grafted chains, and from Rouse-like scalings of the relaxation time with mode number and chain length. The Rouse model however seems inapplicable to the two systems with attraction between the grafted and matrix chains (systems 16 and 17 in Tables 1 and 2), which prevented us from obtaining relaxation times for one of these systems. The model developed here thus represents the viscoelastic moduli of all simulated systems except the above two attractive systems. According to the Rouse model, the storage and loss moduli of pure-component systems composed of matrix chains or grafted chains at the same temperature $T$ and monomer density $c(=0.82$ in this study) as that of the PNCs are given by $^{39}$

$$
\begin{aligned}
& G_{\text {matrix }}^{\prime}(\omega)=\frac{c k_{\mathrm{B}} T}{L_{\text {matrix }}} \sum_{p=1}^{L_{\text {matrix }}} \frac{\omega^{2} \tau_{\text {matrix }, p}{ }^{2}}{1+\omega^{2} \tau_{\text {matrix }, p}^{2}} \\
& G_{\text {matrix }}^{\prime \prime}(\omega)=\frac{c k_{\mathrm{B}} T}{L_{\text {matrix }}} \sum_{p=1}^{L_{\text {matrix }}} \frac{\omega \tau_{\text {matrix }, p}}{1+\omega^{2} \tau_{\text {matrix }, p}^{2}} \\
& G_{\text {graft }}^{\prime \prime}(\omega)=\frac{c k_{\mathrm{B}} T}{L_{\text {graft }}} \sum_{p=1}^{L_{\text {graft }}} \frac{\omega^{2} \tau_{\text {graft }, p}^{2}}{1+\omega^{2} \tau_{\text {graft }, p}^{2}} \\
& G_{\text {graft }}^{\prime \prime}(\omega)=\frac{c k_{\mathrm{B}} T}{L_{\text {graft }}} \sum_{p=1}^{L_{\text {graft }}} \frac{\omega \tau_{\text {graft }, p}}{1+\omega^{2} \tau_{\text {graft }, p}{ }^{2}}
\end{aligned}
$$

The mode relaxation times $\tau_{\text {matrix }, p}$ and $\tau_{\text {graft }, p}$ for the matrix and grafted chains can be related to their respective Rouse times $\tau_{\text {matrix }, \mathrm{R}} \equiv \tau_{\text {matrix }, 1}$ and $\tau_{\text {graft, } \mathrm{R}} \equiv \tau_{\text {graft }, 1}$ via

$$
\tau_{\text {matrix }, p}=\tau_{\text {matrix }, \mathrm{R}}\left[\sin ^{2}\left(\frac{\pi}{2 L_{\text {matrix }}}\right)\right]\left[\sin ^{2}\left(\frac{\pi p}{2 L_{\text {matrix }}}\right)\right]^{-1}
$$

$$
\tau_{\text {graft }, p}=\tau_{\text {graft }, \mathrm{R}}\left[\sin ^{2}\left(\frac{\pi}{4\left(L_{\text {graft }}+1 / 2\right)}\right)\right]\left[\sin ^{2}\left(\frac{\pi(2 p-1)}{4\left(L_{\text {graft }}+1 / 2\right)}\right)\right]^{-1}
$$

Equations 16-28 form the basis of our phenomenological model that allows prediction of the storage and loss moduli of PNCs based on a select few parameters: temperature, monomer density, lengths and Rouse times of the matrix and grafted chains, and volume fractions of the NPs, matrix chains, and grafted chains. At sufficiently low frequencies $\omega$, it is possible to further simplify the above model to yield closed-form analytical expressions for the PNC moduli $G^{\prime}(\omega)$ and $G^{\prime \prime}(\omega)$; the derivation is provided in the Appendix.

To investigate how well the above model captures our simulation results, we compared the $G^{\prime}(\omega)$ and $G^{\prime \prime}(\omega)$ predicted from our model against those computed from our MD simulations. We performed this comparison at two different values of $\omega$ : one in the low frequency regime $(\omega=$ $\left.10^{-5}\right)$ and another in the high frequency regime $(\omega=1)$. In our model predictions, we used the Rouse times computed from the simulations (via eqs 10-13), though they could also be independently obtained from other theoretical arguments. ${ }^{39}$ Figure $10 \mathrm{a}-\mathrm{d}$ shows the two moduli computed at the two
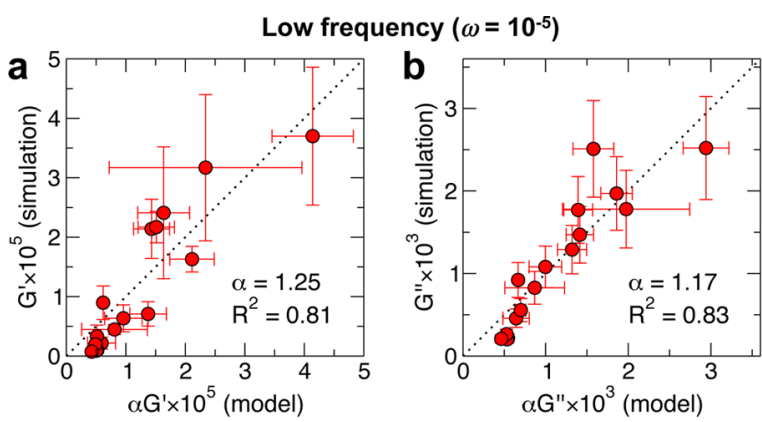

High frequency $(\omega=1)$
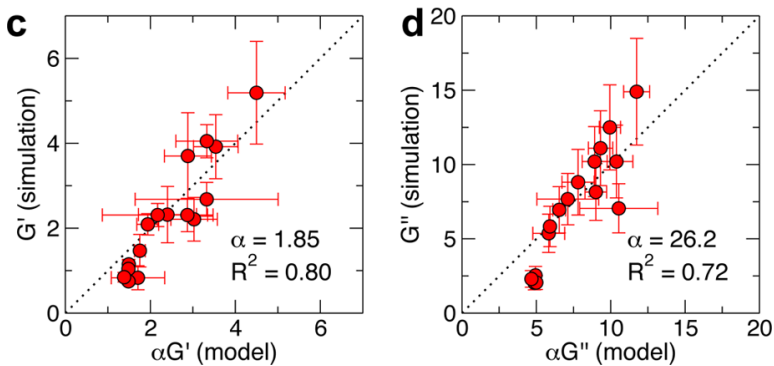

Figure 10. Comparison of the viscoelastic moduli predicted from the phenomenological model against those computed from $\mathrm{MD}$ simulations. The comparison is shown for $G^{\prime}$ and $G^{\prime \prime}$ obtained at low frequency $(a, b)$ and at high frequency $(c, d)$. The dashed lines indicate perfect agreement between model and simulations. The multiplicative constant $\alpha$ and the correlation coefficient $R^{2}$ for each data set are also indicated.

frequencies from MD simulations of all systems listed in Table 1, except two for reasons mentioned earlier, plotted against the corresponding moduli predicted by our model. To permit more uniform comparison across the four data sets, the model predictions in each data set have been scaled by a factor $\alpha$ that maximizes the agreement between the scaled model predictions and the simulation results; i.e., the data points deviate the least from the diagonal line. We find that in all four cases the data fall close to the diagonal, suggesting that the model captures well 
the trends in $G^{\prime}$ and $G^{\prime \prime}$ at both low and high frequencies. That the factor $\alpha$ is close to 1 for both moduli at low frequency (Figure 10a,b) suggests that the model also make remarkably good quantitative predictions of $G^{\prime}$ and $G^{\prime \prime}$ at low frequencies. However, this is not the case at high frequencies (Figure 10c,d), where the model underpredicts the moduli obtained from simulations, which is expecially severe for $G^{\prime \prime}$ where $\alpha \approx 26$. The disagreement at high frequencies is somewhat expected as the Rouse model does not account for relaxation of small groups of bonds and bond angles of the FENE chains used for treating all polymer chains in our MD simulations. Thus, in spite of its simplicity, the model developed here offers reasonable quantitative predictions of the viscoelastic moduli of the simulated PNCs at low frequencies and reasonable qualitative predictions at high frequencies.

The model has potential for further refinement to improve its predictive power. First, a more detailed treatment of the shear distortion effects arising from NP grafts and how these effects enhance the viscoelastic moduli of the matrix polymer is required. Currently, we assume that the grafts distort the strain field similarly to the NPs, i.e., by preventing slippage of matrix chains at their interface with the grafted chains, which we account for by replacing the volume fraction of the NPs in existing models (eq 18) with that of the NPs plus the grafted chains. Intuitively, one would expect the strain-distortion effect from the flexible grafted chains to be weaker than that arising from the rigid cores of the NPs due to the ability of the grafts to relax the strain field through conformational changes. However, the grafted chains introduce other effects that could conceivably lead to additional shear distortion. For instance, the grafted chains have a long reach into the polymer that allows NPs to form intermittent, percolating networks. Hydrodynamic interactions between the grafted chains due to their close vicinity to each other and NP surface corrugation due to some chains being extended and others collapsed could also lead to additional strain distortion effects. Such effects could potentially add up to make the effective shear distortion from flexible grafted chains comparable to those from rigid bodies. Clearly, more theoretical and simulation studies are required to fully understand and model these effects. Also, contrary to our assumption in eqs 16 and 17, the moduli enhancement from shear distortion could also depend on frequency.

Second, we use simple mixture rules to estimate the properties of a polymer mixture from properties of individual polymer species. While this strategy works well at the macroscopic level, the applicability of these rules is unclear for nanoscale systems where the interface thickness is on the order of the phases themselves. It is likely that a separate treatment of the interface, similar to that of the individual phases, might be required. Third, we use a Rouse model of endtethered chains (only one end fixed) to obtain the relaxation times of our grafted chains. However, additional studies are required to investigate how the presence of an impenetrable surface at the tethering point, such as the curved NP surface in our systems, affects the relaxation time of the chains. Finally, our model hinges on the Rouse theory, which may not be quite applicable for some polymeric systems, such as melts of long polymer chains that exhibit significant entanglements or solutions of polymer chains where hydrodynamic interactions between polymer segments become important. In these cases, the tube model ${ }^{54}$ that accounts for polymer entanglement and the Zimm model ${ }^{55}$ that accounts for hydrodynamic interactions might be more applicable.

\section{CONCLUSIONS}

We have investigated the viscoelastic properties of PNCs composed of polymer-grafted NPs distributed within a polymer matrix. Specifically, we have carried out equilibrium MD simulations of coarse-grained models of the PNCs and computed their frequency-dependent storage and loss moduli. We find that the PNCs containing grafted NPs exhibit significantly higher $G^{\prime}(\omega)$ and $G^{\prime \prime}(\omega)$ across all frequencies examined compared to those containing bare NPs. We show that most of this enhancement arises from the additional distortion of shear field in the polymer matrix caused by the addition of NP grafts into the matrix. The remaining enhancement at low frequencies arises from the slower relaxation times of the NP grafts compared to the matrix chains, especially when the grafts are at least half as long as the matrix chains. We have also examined the effects of a number of NP-associated parameters on $G^{\prime}(\omega)$ and $G^{\prime \prime}(\omega)$. Our results indicate that both moduli decrease with increasing NP size and increase with increasing NP loading, graft length, grafting density, and graft/matrix affinity, with varying sensitivities to each of the parameters. Furthermore, the parameters are found to affect $G^{\prime}(\omega)$ and $G^{\prime \prime}(\omega)$ in distinct manners, with some effects being largely frequency-independent, others being largely frequency-dependent, and yet others showing mixed behavior. We have provided suitable explanations for each of these effects based on shear distortion, relaxation times of the matrix and grafted chains, and radial density profiles. Toward the end, we propose a phenomenological model, based on Rouse theory, that captures reasonably well the above parametric trends and, in fact, yields quantitiative predictions of the storage and loss moduli at low frequencies. Several possible future extensions of this model are also discussed. The results presented here should be useful to polymer experimentalists studying rheological behavior of PNCs and designing them for specific applications as well as polymer physicists aiming to understand and model the rich rheological behavior of PNCs.

\section{APPENDIX}

At sufficiently low frequencies, it is possible to further simplify the phenomenological model developed here (eqs 16-28) to yield simple closed-form analytical estimates of $G^{\prime}(\omega)$ and $G^{\prime \prime}(\omega)$. The low-frequency response is arguably more relevant to practical applications of PNCs than the high-frequency response. Specifically, in the limits $\omega \ll 1 / \tau_{\text {matrix,R }}$ and $\ll 1 /$ $\tau_{\text {graft, } R}$ eqs $23-26$ can be reduced to the following simpler expressions:

$$
\begin{aligned}
& G_{\text {matrix }}^{\prime}(\omega) \approx \frac{c k_{\mathrm{B}} T \omega^{2}}{L_{\text {matrix }}} \sum_{p=1}^{L_{\text {matrix }}} \tau_{\text {matrix }, p}{ }^{2} \approx \frac{c k_{\mathrm{B}} T \omega^{2} \tau_{\text {matrix }, \mathrm{R}}{ }^{2}}{L_{\text {matrix }}} \times \\
& \sum_{p=1}^{\infty} \frac{1}{p^{4}}=\frac{\pi^{4}}{90} \frac{c k_{\mathrm{B}} T \omega^{2} \tau_{\text {matrix }, \mathrm{R}}^{2}}{L_{\text {matrix }}} \\
& G_{\text {matrix }}^{\prime \prime}(\omega) \approx \frac{c k_{\mathrm{B}} T \omega}{L_{\text {matrix }}} \sum_{p=1}^{L_{\text {matrix }}} \tau_{\text {matrix }, p} \approx \frac{c k_{\mathrm{B}} T \omega \tau_{\text {matrix }, \mathrm{R}}}{L_{\text {matrix }}} \sum_{p=1}^{\infty} \frac{1}{p^{2}} \\
& =\frac{\pi^{2}}{6} \frac{c k_{\mathrm{B}} T \omega \tau_{\text {matrix }, \mathrm{R}}}{L_{\text {matrix }}}
\end{aligned}
$$




$$
\begin{gathered}
G_{\text {graft }}^{\prime}(\omega) \approx \frac{c k_{\mathrm{B}} T \omega^{2}}{L_{\text {graft }}} \sum_{p=1}^{L_{\text {graft }}} \tau_{\text {graft }, p}{ }^{2} \approx \frac{c k_{\mathrm{B}} T \omega^{2} \tau_{\text {graft }, \mathrm{R}}{ }^{2}}{L_{\text {graft }}} \times \\
\sum_{p=1}^{\infty} \frac{1}{(2 p-1)^{4}}=\frac{\pi^{4}}{96} \frac{c k_{\mathrm{B}} T \omega^{2} \tau_{\text {graft } \mathrm{R}}{ }^{2}}{L_{\text {graft }}} \\
G_{\text {graft }}^{\prime \prime}(\omega) \approx \frac{c k_{\mathrm{B}} T \omega}{L_{\text {graft }}} \sum_{p=1}^{L_{\text {graft }}} \tau_{\text {graft }, p} \approx \frac{c k_{\mathrm{B}} T \omega \tau_{\text {graft } \mathrm{R}}}{L_{\text {graft }}} \times \\
\sum_{p=1}^{\infty} \frac{1}{(2 p-1)^{2}}=\frac{\pi^{2}}{8} \frac{c k_{\mathrm{B}} T \omega \tau_{\text {graft } \mathrm{R}}}{L_{\text {graft }}}
\end{gathered}
$$

To obtain the above simplifications, we used the following approximations of eqs 27 and 28: $\tau_{\text {matrix }, p} \approx \tau_{\text {matrix, } \mathrm{R}} / p^{2}$ and $\tau_{\text {graft, } p}$ $\approx \tau_{\text {graft, } \mathrm{R}} /(2 p-1)^{2}$. While the above approximations are valid only at small $p$, their failure at large $p$ affects the results only marginally as the fast modes at large $p$ contribute negligibly to the moduli. This negligible contribution from the higher modes also allows us to cast the finite series in eqs 29-32 as infinite series, which then allows us to obtain their sums in terms of known Riemman zeta functions. ${ }^{56}$

Substituting eqs 29-32 into eqs 21 and 22 and the resulting expression along with eq 18 into eqs 16 and 17 yields the following analytical form for the storage and loss modulus of PNCs at low frequencies:

$$
\begin{aligned}
& G^{\prime}(\omega)=\frac{\pi^{4} c k_{\mathrm{B}} T \omega^{2} \tau_{\text {matrix, } \mathrm{R}}{ }^{2} \tau_{\text {graft, } \mathrm{R}}{ }^{2}\left[1+2.5\left(\phi_{\mathrm{NP}}+\phi_{\text {graft }}\right)\right]}{96 \phi_{\text {graft }} L_{\text {graft }} \tau_{\text {matrix }, \mathrm{R}}{ }^{2}+90 \phi_{\text {matrix }} L_{\text {matrix }} \tau_{\text {graft, } \mathrm{R}}{ }^{2}} \\
& G^{\prime \prime}(\omega)=\frac{\pi^{2} c k_{\mathrm{B}} T \omega \tau_{\text {matrix } \mathrm{R}} \tau_{\text {graft }, \mathrm{R}}\left[1+2.5\left(\phi_{\mathrm{NP}}+\phi_{\text {graft }}\right)\right]}{8 \phi_{\text {graft }} L_{\text {graft }} \tau_{\text {matrix } \mathrm{R}}+6 \phi_{\text {matrix }} L_{\text {matrix }} \tau_{\text {graft, } \mathrm{R}}}
\end{aligned}
$$

yielding the characteristic $\omega^{2}$ and $\omega$ scalings of the storage and loss modulus observed at low frequencies.

\section{ASSOCIATED CONTENT}

\section{S Supporting Information}

Discussions on NP aggregation, sampling exhaustiveness, and attractive site energetics. Tables of chain radius of gyration, normalized grafting density, NP diffusion coefficient, and NP rotational relaxation time. Plots of inter-particle and inter-bead radial distribution functions, Rouse time versus mode number, storage and loss modulus of pure polymer and of PNCs as a function of NP loading and size, and radial monomer density profiles as a function of grafted chain length and grafting density. This material is available free of charge via the Internet at http://pubs.acs.org.

\section{AUTHOR INFORMATION}

\section{Corresponding Author}

*E-mail garya@ucsd.edu; Ph 858-822-5542; Fax 858-534-9553 (G.A.).

\section{Notes}

The authors declare no competing financial interest.

\section{ACKNOWLEDGMENTS}

The authors gratefully acknowledge support from the Office of Naval Research under Grant N00014-09-1-1126 to University of California, San Diego. Acknowledgment is also made to the donors of the American Chemical Society Petroleum Research Fund (Award No. 52515-ND7) for partial support of this research. The authors also thank Dr. Bedri Arman for preliminary simuations of polymer nanocomposites, Prof. Jay Oswald for providing computational resources, and Prof. Sia Nemat-Nasser, Prof. Zhibin Guan, and Dr. Roshdy Barsoum for valuable discussions.

\section{REFERENCES}

(1) Jancar, J.; Douglas, J. F.; Starr, F. W.; Kumar, S. K.; Cassagnau, P.; Lesser, A. J.; Sternstein, S. S.; Buehler, M. J. Polymer 2010, 51, 33213343.

(2) Green, P. F.; Oh, H.; Akcora, P.; Kumar, S. K. Structure and Dynamics of Polymer Nanocomposites Involving Chain-Grafted Spherical Nanoparticles. In Dynamics of Soft Matter: Neutron Applications; Garca Sakai, V., Alba-Simionesco, C., Chen, S.-H., Eds.; Neutron Scattering Applications and Techniques; Springer: Boston, MA, 2012.

(3) Filippone, G.; Acierno, D. Nanoparticle Dynamics in Polymer Melts. In Smart Nanoparticles Technology; Hashim, A., Ed.; InTech: Rijeka, Croatia, 2012; Vol. V.

(4) Witten, T. A.; Pincus, P. A. Macromolecules 1986, 19, 2509-2513.

(5) Gao, B.; Arya, G.; Tao, A. R. Nat. Nanotechnol. 2012, 7, 433-437.

(6) Zou, H.; Wu, S.; Shen, J. Chem. Rev. 2008, 108, 3893-3957.

(7) Tanaka, T.; Montanari, G. C.; Mulhaupt, R. IEEE Trans. Dielectr. Electr. Insul. 2004, 11, 763-784.

(8) Alexandre, M.; Dubois, P. Mater. Sci. Eng., R 2000, 28, 1-63.

(9) McNally, T.; Pötschke, P.; Halley, P.; Murphy, M.; Martin, D.; Bell, S. E.; Brennan, G. P.; Bein, D.; Lemoine, P.; Quinn, J. P. Polymer 2005, 46, 8222-8232.

(10) Zhang, Q.; Archer, L. A. Langmuir 2002, 18, 10435-10442.

(11) Ren, J.; Silva, A. S.; Krishnamoorti, R. Macromolecules 2000, 33, 3739-3746.

(12) Krishnamoorti, R.; Giannelis, E. P. Macromolecules 1997, 9297, 4097-4102.

(13) Galgali, G.; Ramesh, C.; Lele, A. Macromolecules 2001, 34, 852858.

(14) Akcora, P.; Kumar, S. K.; Moll, J.; Lewis, S.; Schadler, L. S.; Li, Y.; Benicewicz, B. C.; Sandy, A.; Narayanan, S.; Ilavsky, J.; Thiyagarajan, P.; Colby, R. H.; Douglas, J. F. Macromolecules 2010, 43, 1003-1010.

(15) Sen, S.; Thomin, J. D.; Kumar, S. K.; Keblinski, P. Macromolecules 2007, 40, 4059-4067.

(16) Chevigny, C.; Dalmas, F.; Cola, E. D.; Gigmes, D.; Bertin, D.; Boue, F.; Jestin, J. Macromolecules 2011, 44, 122-133.

(17) Pryamitsyn, V.; Ganesan, V. Macromolecules 2006, 39, 844-856.

(18) Termonia, Y. J. Polym. Sci., Part B: Polym. Phys. 2010, 48, 687692.

(19) Smith, G. D.; Bedrov, D.; Li, L.; Byutner, O. J. Chem. Phys. 2002, 117, 9478-9489.

(20) Guth, E. J. Appl. Phys. 1945, 16, 20-25.

(21) Einstein, A. Ann. Phys. 1906, 19, 289-306.

(22) Yan, L.-T.; Xie, X.-M. Prog. Polym. Sci. 2012, 1-37.

(23) Hall, L. M.; Jayaraman, A.; Schweizer, K. S. Curr. Opin. Solid State Mater. Sci. 2010, 14, 38-48.

(24) Ganesan, V.; Jayaraman, A. Soft Matter 2014, 10, 13-38.

(25) Kremer, K.; Grest, G. S. J. Chem. Phys. 1990, 92, 5057-5086.

(26) Weeks, J. D.; Chandler, D.; Anderson, H. C. J. Chem. Phys. $1971,54,5237-5247$.

(27) Rakhmanov, E.; Saff, E.; Zhou, Y. Math. Res. Lett. 1994, 1, 647662.

(28) Saff, E. B.; Kuijlaars, A. B. Math. Intell. 1997, 19, 5-11.

(29) Holzworth, K.; Williams, G.; Arman, B.; Guan, Z.; Arya, G.; Nemat-Nasser, S. Polyurea With Hybrid Polymer Grafted Nanoparticles: A Parametric Study. ASME 2012 International Mechanical Engineering Congress and Exposition, 2012. 
(30) Arman, B.; Reddy, A. S.; Arya, G. Macromolecules 2012, 45, 3247-3255.

(31) Agrawal, V.; Arya, G.; Oswald, J. Macromolecules 2014, 47, 3378-3389.

(32) Arya, G.; Rottler, J.; Panagiotopoulos, A. Z.; Srolovitz, D. J.; Chaikin, P. M. Langmuir 2005, 21, 11518-11527.

(33) Plimpton, S. J. Comput. Phys. 1995, 117, 1-42.

(34) Nose, S. Prog. Theor. Phys. Supp. 1991, 103, 1-46.

(35) Trombly, D. M.; Ganesan, V. J. Chem. Phys. 2010, 133, 154904.

(36) Kumar, S. K.; Jouault, N.; Benicewicz, B.; Neely, T. Macromolecules 2013, 46, 3199-3214.

(37) Meng, D.; Kumar, S. K.; Lane, J. M. D.; Grest, G. S. Soft Matter 2012, 8, 5002-5010.

(38) Likhtman, A. E. Viscoelasticity and Molecular Rheology. In Polymer Science: A Comprehensive Reference; Matyjaszewski, K., Mšller, M., Eds.; Elsevier BV: Amsterdam, 2012; Vol. 1.

(39) Doi, M.; Edwards, S. F. The Theory of Polymer Dynamics; Clarendon Press: Oxford, 1994.

(40) Marciano, Y.; Brochard-Wyart, F. Macromolecules 1995, 28, 985-990.

(41) Quake, S. R.; Babcock, H.; Chu, S. Nature 1997, 388, 151-154.

(42) Einstein, A. Ann. Phys. 1911, 591-592.

(43) Eilers, V. H. Kolloid-Z. 1941, 3, 313-321.

(44) Wildemuth, C. R.; Wiliams, M. C. Rheol. Acta 1984, 635, 627635.

(45) Chao, H.; Riggleman, R. A. Polymer 2013, 54, 5222-5229.

(46) Toepperwein, G. N.; Riggleman, R. A.; de Pablo, J. J. Macromolecules 2012, 45, 543-554.

(47) Chremos, A.; Panagiotopoulos, A. Z.; Koch, D. L. J. Chem. Phys. 2012, 136, 044902.

(48) Goyal, S.; Escobedo, F. A. J. Chem. Phys. 2011, 135, 184902.

(49) Fu, S.-Y.; Feng, X.-Q.; Lauke, B.; Mai, Y.-W. Composites, Part B 2008, 39, 933-961.

(50) Ji, X. L.; Jing, J. K.; Jiang, W.; Jiang, B. Z. Polym. Eng. Sci. 2002, 42, 983-993.

(51) Douce, J.; Boilot, J.-P.; Biteau, J.; Scodellaro, L.; Jimenez, A. Thin Solid Films 2004, 466, 114-122.

(52) Green, P. F. Soft Matter 2011, 7, 7914-7926.

(53) Ahmed, S.; Jones, F. R. J. Mater. Sci. 1990, 25, 4933-4942.

(54) de Gennes, P.-G. J. Chem. Phys. 1971, 55, 572-579.

(55) Zimm, B. H. J. Chem. Phys. 1956, 24, 269-278.

(56) Abramowitz, M.; Stegun, I. A. Handbook of Mathematical Functions; Dover: New York, 1972. 